\title{
BAT AGN Spectroscopic Survey. XI. The Covering Factor of Dust and Gas in Swift/BAT Active Galactic Nuclei
}

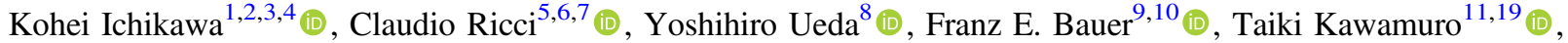 \\ Michael J. Koss ${ }^{12}\left(\mathbb{D}\right.$, Kyuseok $\mathrm{Oh}^{8,19}$ (D), David J. Rosario ${ }^{13}\left(\mathbb{D}\right.$, T. Taro Shimizu ${ }^{14}\left(\mathbb{D}\right.$, Marko Stalevski ${ }^{15,16}\left(\mathbb{0}\right.$, Lindsay Fuller ${ }^{2}$, \\ Christopher Packham ${ }^{2,11}$, and Benny Trakhtenbrot ${ }^{17,18}$ (1) \\ ${ }^{1}$ Department of Astronomy, Columbia University, 550 West 120th Street, New York, NY 10027, USA; k.ichikawa@astr.tohoku.ac.jp \\ ${ }^{2}$ Department of Physics and Astronomy, University of Texas at San Antonio, One UTSA Circle, San Antonio, TX 78249, USA \\ ${ }^{3}$ Frontier Research Institute for Interdisciplinary Sciences, Tohoku University, Sendai, Miyagi 980-8578, Japan \\ ${ }^{4}$ Astronomical Institute, Tohoku University, Aramaki, Aoba-ku, Sendai, Miyagi 980-8578, Japan \\ ${ }^{5}$ Núcleo de Astronomía de la Facultad de Ingeniería, Universidad Diego Portales, Av. Ejército Libertador 441, Santiago, Chile \\ ${ }^{6}$ Kavli Institute for Astronomy and Astrophysics, Peking University, Beijing 100871, People's Republic of China \\ ${ }^{7}$ Chinese Academy of Sciences South America Center for Astronomy, Camino El Observatorio 1515, Las Condes, Santiago, Chile \\ ${ }^{8}$ Department of Astronomy, Kyoto University, Oiwake-cho, Sakyo-ku, Kyoto 606-8502, Japan \\ ${ }^{9}$ Millennium Institute of Astrophysics (MAS), Nuncio Monseñor Sótero Sanz 100, Providencia, Santiago, Chile \\ ${ }^{10}$ Space Science Institute, 4750 Walnut Street, Suite 205, Boulder, CO 80301, USA \\ ${ }^{11}$ National Astronomical Observatory of Japan, 2-21-1 Osawa, Mitaka, Tokyo 181-8588, Japan \\ ${ }^{12}$ Eureka Scientific, 2452 Delmer Street Suite 100, Oakland, CA 94602-3017, USA \\ ${ }^{13}$ Centre for Extragalactic Astronomy, Department of Physics, Durham University, South Road, DH1 3LE Durham, UK \\ ${ }^{14}$ Max-Planck-Institut für extraterrestrische Physik, Postfach 1312, D-85741, Garching, Germany \\ ${ }^{15}$ Astronomical Observatory, Volgina 7, 11060 Belgrade, Serbia \\ ${ }^{16}$ Sterrenkundig Observatorium, Universiteit Gent, Krijgslaan 281-S9, Gent B-9000, Belgium \\ ${ }^{17}$ Department of Physics, ETH Zurich, Wolfgang-Pauli-Strasse 27, CH-8093 Zurich, Switzerland \\ ${ }^{18}$ School of Physics and Astronomy, Tel Aviv University, Tel Aviv 69978, Israel \\ Received 2018 March 7; revised 2018 October 12; accepted 2018 November 2; published 2019 January 2
}

\begin{abstract}
We quantify the luminosity contribution of active galactic nuclei (AGNs) to the $12 \mu$ m, mid-infrared (MIR; 5-38 $\mu \mathrm{m})$, and total IR $(5-1000 \mu \mathrm{m})$ emission in the local AGNs detected in the all-sky 70 month Swift/Burst Alert Telescope (BAT) ultrahard X-ray survey. We decompose the IR spectral energy distributions (SEDs) of 587 objects into the AGN and starburst components using templates for an AGN torus and a star-forming galaxy. This enables us to recover the emission from the AGN torus including the low-luminosity end, down to $\log \left(L_{14-150} / \mathrm{erg} \mathrm{s}^{-1}\right) \simeq 41$, which typically has significant host galaxy contamination. The sample demonstrates that the luminosity contribution of the AGN to the $12 \mu \mathrm{m}$, the MIR, and the total IR bands is an increasing function of the 14-150 keV luminosity. We also find that for the most extreme cases, the IR pure-AGN emission from the torus can extend up to $90 \mu \mathrm{m}$. The total IR AGN luminosity obtained through the IR SED decomposition enables us to estimate the fraction of the sky obscured by dust, i.e., the dust covering factor. We demonstrate that the median dust covering factor is always smaller than the median X-ray obscuration fraction above an AGN bolometric luminosity of $\log \left(L_{\mathrm{bol}}^{(\mathrm{AGN})} / \mathrm{erg} \mathrm{s}^{-1}\right) \simeq 42.5$. Considering that the X-ray obscuration fraction is equivalent to the covering factor coming from both the dust and gas, this indicates that an additional neutral gas component, along with the dusty torus, is responsible for the absorption of X-ray emission.
\end{abstract}

Key words: galaxies: active - galaxies: nuclei - infrared: galaxies

Supporting material: figure set, machine-readable table

\section{Introduction}

One of the fundamental open questions of extragalactic astrophysics is how supermassive black holes (SMBHs) and their host galaxies coevolve (e.g., Alexander \& Hickox 2012). Active galactic nuclei (AGNs) are the best targets for understanding this process of coevolution, because they are in the stage where mass accretion onto SMBHs occurs with the release of large amounts of radiation (e.g., Yu \& Tremaine 2002; Marconi et al. 2004), until they reach their maximum achievable mass of $M_{\mathrm{BH}} \simeq 10^{10.5} M_{\odot}$ (Netzer 2003; McLure \& Dunlop 2004; Trakhtenbrot 2014; Jun et al. 2015; Inayoshi \& Haiman 2016; Ichikawa \& Inayoshi 2017).

Ultrahard $(E>10 \mathrm{keV})$ X-ray observations are one of the most reliable methods for identifying AGNs. Thanks to the

19 JSPS fellow. combination of (1) a strong penetration power up to $\log \left(N_{\mathrm{H}} / \mathrm{cm}^{-2}\right) \simeq 24$ (e.g., Ricci et al. 2015) and (2) the high contrast with stellar X-ray emission (e.g., Mineo et al. 2012), ultrahard X-ray surveys allow an unbiased census of AGNs up to Compton-thick levels (e.g., Koss et al. 2016). Among the recent available surveys, Swift/BAT provides the most sensitive X-ray survey of the whole sky in the $14-195 \mathrm{keV}$ range, reaching a flux level of (1.0-1.3) $\times 10^{-11} \mathrm{erg} \mathrm{s}^{-1} \mathrm{~cm}^{-2}$ in the first 70 months of operations (Baumgartner et al. 2013), and a deeper flux of $(7.2-8.4) \times 10^{-12} \mathrm{erg} \mathrm{s}^{-1} \mathrm{~cm}^{-2}$ in the recently updated 105 month catalog (Oh et al. 2018).

Infrared (IR) observations also provide an effective method to study AGNs because the central engine of an AGN is expected to be surrounded by a dusty "torus" (Krolik \& Begelman 1986), which is heated by the AGN and re-emits thermally in the mid-IR (MIR) (e.g., Gandhi et al. 2009; 
Ichikawa et al. 2012, 2017; Asmus et al. 2015). A recent upward revision of black hole scaling relations (Kormendy \& Ho 2013) indicates that the local mass density in black holes should be higher, suggesting that a larger population of heavily obscured AGN gas and dust is required to fill the mass gap of the revised local black hole mass density (e.g., Novak 2013; Comastri et al. 2015). These populations contribute significantly to the infrared background (e.g., Murphy et al. 2011; Delvecchio et al. 2014), especially in the MIR band (Risaliti et al. 2002). However, since star formation from the host galaxy sometimes contaminates the MIR emission, especially for low-luminosity AGNs with $L_{14-150}<10^{43} \mathrm{erg} \mathrm{s}^{-1}$ (e.g., Ichikawa et al. 2017), and the torus is too compact $(<10 \mathrm{pc}$; e.g., Jaffe et al. 2004) to be fully resolved (e.g., García-Burillo et al. 2016; Imanishi et al. 2018), the precise estimation of AGN thermal activity is not straightforward.

Fortunately, several methods have been proposed to isolate the emission from the torus from the starburst component. One of them is to use MIR observations with high spatial resolution ( $\sim$ !. 3-0.! 7) to resolve the starburst emission of the host galaxies down to scales of $10 \mathrm{pc}$ (Packham et al. 2005; Radomski et al. 2008; Hönig et al. 2010; Alonso-Herrero et al. 2011, 2016; Ramos Almeida et al. 2011; González-Martín et al. 2013; Asmus et al. 2014; Ichikawa et al. 2015; MartínezParedes et al. 2017). In addition, the advent of IR interferometric observations, with their exquisite resolving power (with baselines up to $130 \mathrm{~m}$ ), has spatially resolved the dusty nuclear regions and shown that their outer radii in the MIR are typically several parsecs (e.g., Jaffe et al. 2004; Raban et al. 2009; Burtscher et al. 2013). Notably, some show the polar elongated dust emission suggestive of a dusty outflow (Hönig et al. 2012, 2013; Tristram et al. 2014; López-Gonzaga et al. 2016, Leftley et al. 2018). However, because of the limited sensitivity and the spatial resolution of current telescopes (see a recent review by Burtscher et al. 2016), these two methods are available only for a few tens of bright sources located in the very local universe $(z<0.01)$. Another possible approach is to separate the spectral emission of the AGN and the starburst (SB) component. Multiple decomposition methods have been applied to MIR spectra, mainly using aromatic features as a proxy for star formation (e.g., Tran et al. 2001; Lutz et al. 2004; Sajina et al. 2007; Alonso-Herrero et al. 2012; Ichikawa et al. 2014; Hernán-Caballero et al. 2015; Kirkpatrick et al. 2015; Symeonidis et al. 2016), to broadband IR spectral energy distributions (SEDs, e.g., da Cunha et al. 2008; Hatziminaoglou et al. 2008; Xu et al. 2015; Lyu et al. 2016, 2017; Shimizu et al. 2017), and to the combination of both spectra and SEDs (e.g., Mullaney et al. 2011). The advantage of the SED decomposition is that it is less affected by the differing spatial resolutions inherent in aperture photometry, and can be applied to high- $z$ sources (e.g., Stanley et al. 2015; Lyu et al. 2016; Mateos et al. 2016) and/or to large $(N>100)$ samples, for which MIR imaging and spectroscopy with high spatial resolution would require significant amounts of time on large-diameter $(>8 \mathrm{~m})$ telescopes.

In this paper, we decompose the IR SEDs of the ultrahard X-ray-selected Swift/BAT 70 month AGN catalog (Baumgartner et al. 2013) into AGN and host galaxy components. Thanks to intensive follow-up observations by BAT AGN Spectroscopic Survey $^{20}$ (BASS; Koss et al. 2017; Lamperti et al. 2017;

${ }^{20}$ www.bass-survey.com
Ricci et al. 2017b), we are able to obtain reliable information on the gas column density $\left(N_{\mathrm{H}}\right)$, absorption-corrected $14-150 \mathrm{keV}$ X-ray luminosity, and black hole mass $\left(M_{\mathrm{BH}}\right)$ of the sample.

The main goal of this work is to quantitatively assess the AGN contribution to the $12 \mu \mathrm{m}$ band, MIR (5-38 $\mu \mathrm{m})$ band, and total IR $(5-1000 \mu \mathrm{m})$ band down to $\log \left(L_{14-150} / \mathrm{erg} \mathrm{s}^{-1}\right) \simeq 41$ in order to investigate torus (1) the correlation between MIR and X-ray luminosity and (2) the dust covering factor of the torus, minimizing issues related to host galaxy contamination. Throughout the paper, we adopt standard cosmological parameters $\left(H_{0}=70.0 \mathrm{~km} \mathrm{~s}^{-1} \mathrm{Mpc}^{-1}, \Omega_{\mathrm{M}}=0.3\right.$, and $\left.\Omega_{\Lambda}=0.7\right)$.

\section{Sample}

Our initial sample is based on the sample of Ichikawa et al. (2017), which contains the 606 non-blazar AGNs from the Swift/BAT 70 month catalog (Baumgartner et al. 2013) at galactic latitudes $\left(|b|>10^{\circ}\right)$ for which secure spectroscopic redshifts are available. In this study, we use the column density $\left(N_{\mathrm{H}}\right)$ and the absorption-corrected $14-150 \mathrm{keV}$ luminosity $\left(L_{14-150}\right)$ tabulated in Ricci et al. (2017b). They are also summarized in Table 1.

In Ichikawa et al. (2017), we reported the 3-500 $\mu \mathrm{m} \mathrm{IR}$ counterparts for our AGN sample, utilizing the IR catalogs obtained from WISE (Wright et al. 2010; Cutri et al. 2013), AKARI (Murakami et al. 2007), IRAS (Beichman et al. 1988), and Herschel (Griffin et al. 2010; Poglitsch et al. 2010). Out of the 606 sources, we identified 604, 560, 601, and 402 counterparts in the total IR, near-IR (NIR; $<5 \mu \mathrm{m}$ ), MIR, and far-IR (FIR; 60-500 $\mu \mathrm{m}$ ) bands, respectively. The reader should refer to Ichikawa et al. (2017) for details of the IR catalogs. While Ichikawa et al. (2017) compiled the representative fluxes at $12,22,70$, and $90 \mu \mathrm{m}$, by combining similar wavelength bands in the multiple IR catalogs listed above, in this study we regard each IR band with a different central wavelength as independent photometry. Therefore, there are at most 17 available IR photometric bands between 3 and $500 \mu \mathrm{m}$, as identified in Table 1 . For the data points with the same wavelengths (i.e., 12, 25, 60, 100, and $160 \mu \mathrm{m}$ ), the adopted photometry was chosen based on the priorities reported in the IR catalog of Ichikawa et al. (2017) to measure the IR emission from both nucleus and host galaxy in a uniform way for the entire AGN sample. The $12 \mu \mathrm{m}$ flux density was obtained with the following priority: WISE, IRAS/Point Source Catalog (PSC), and IRAS/Faint Source Catalog (FSC). For the 25, 60, and $100 \mu \mathrm{m}$ flux densities, on the other hand, we followed a different order (IRAS/PSC and IRAS/FSC), while for the $160 \mu \mathrm{m}$ flux density we used Herschel/PACS and, when not available, AKARI/FIS. The corrected data are obtained from a wide range of different angular resolutions from Herschel/PACS (70 $\mu \mathrm{m} ; 6$ arcsec) to IRAS/FIR $(100 \mu \mathrm{m} ; \approx 1$ arcmin). Using nearly the same sample, Mushotzky et al. (2014) already showed that the bulk of PACS $70 \mu \mathrm{m}$ is point-like at the spatial resolution of Herschel, suggesting that the FIR emission from the host galaxy is really compact (with a median value of $2 \mathrm{kpc}$ FWHM) and unresolvable for most of our sample. Thus, we conclude that the aperture dependence with more moderate resolutions obtained by $A K A R I$ and IRAS is negligible (see also Meléndez et al. 2014; García-González et al. 2016a; Ichikawa et al. 2017; Lutz et al. 2018). 
Table 1

Column Descriptions for the IR Catalog of the Swift/BAT 70 Month AGN Survey

\begin{tabular}{|c|c|c|c|c|}
\hline Col. \# & Header Name & Format & Unit & Description \\
\hline 1 & objID & string & $\cdots$ & Swift/BAT ID as shown in Baumgartner et al. (2013) \\
\hline 2 & ctpt1 & string & $\cdots$ & optical counterpart name \\
\hline 3 & $z$ & float & $\cdots$ & redshift \\
\hline 5 & lbat_log & float & $\cdots$ & absorption-corrected logarithmic $14-150 \mathrm{keV}$ luminosity $\left(\log \left(L_{14-150} / \mathrm{erg} \mathrm{s}^{-1}\right)\right)$ \\
\hline 6 & lbol_const_log & float & $\cdots$ & logarithmic bolometric AGN luminosity $\left(\log \left(L_{\text {bol }}^{(\mathrm{AGN})} / \mathrm{erg} \mathrm{s}^{-1}\right)\right)$ \\
\hline 7 & lbol_log & float & $\cdots$ & logarithmic bolometric AGN luminosity $\left(\log \left(L_{\mathrm{bol}}^{(\mathrm{M} 04)} / \mathrm{erg} \mathrm{s}^{-1}\right)\right)$ using Marconi et al. (2004) \\
\hline $10(11)$ & fnu4p6_(err)_fqualmod & float & Jy & $4.6 \mu \mathrm{m}$ profile-fitting flux density (error) obtained from WISE \\
\hline $12(13)$ & fnu9a_(err)_fqualmod & float & Jy & $9.0 \mu \mathrm{m}$ flux density (error) obtained from $A K A R I / \mathrm{IRC}$ \\
\hline $14(15)$ & fnu12wipf_(err)_fqualmod & float & Jy & $12 \mu \mathrm{m}$ flux density (error) \\
\hline 16 & fnu12wipcatalog & string & $\cdots$ & reference catalogs for $12 \mu \mathrm{m}: \mathrm{W}=W I S E, \mathrm{Ip}=I R A S / \mathrm{PSC}$, If $=I R A S / \mathrm{FSC}$ \\
\hline $17(18)$ & fnu18a_(err)_fqualmod & float & Jy & $18.0 \mu \mathrm{m}$ flux density (error) obtained from $A K A R I$ \\
\hline $19(20)$ & fnu22w_(err)_fqualmod & float & Jy & $22 \mu \mathrm{m}$ profile-fitting flux density (error) obtained from WISE \\
\hline $29(30)$ & fnu70p_(err)_fqualmod & float & Jy & $70 \mu$ m flux density (error) obtained from Herschel/PACS \\
\hline $31(32)$ & fnu90a_(err)_fqualmod & float & Jy & $90 \mu \mathrm{m}$ flux density (error) obtained from $A K A R I /$ FIS \\
\hline $33(34)$ & fnu100ipf_(err)_fqualmod & float & Jy & $100 \mu \mathrm{m}$ flux density (error) \\
\hline 35 & fnu100ipfcatalog & string & $\cdots$ & reference catalogs for $100 \mu \mathrm{m}:$ Ip $=I R A S / \mathrm{PSC}$, If $=I R A S / \mathrm{FSC}$ \\
\hline $36(37)$ & fnu140a_(err)_fqualmod & float & Jy & $140 \mu \mathrm{m}$ flux density (error) obtained from $A K A R I /$ FIS \\
\hline $38(39)$ & fnu160pa_(err)_fqualmod & float & Jy & $160 \mu \mathrm{m}$ flux density (error) \\
\hline 40 & fnu160pacatalog & string & $\cdots$ & reference catalogs for $160 \mu \mathrm{m}: \mathrm{P}=$ Herschel $/ \mathrm{PACS}, \mathrm{A}=A K A R I / \mathrm{FIS}$ \\
\hline $41(42)$ & fnu250s_(err)_fqualmod & float & Jy & $250 \mu \mathrm{m}$ flux density (error) obtained from Herschel/SPIRE \\
\hline $43(44)$ & fnu350s_(err)_fqualmod & float & Jy & $350 \mu \mathrm{m}$ flux density (error) obtained from Herschel/SPIRE \\
\hline $45(46)$ & fnu500s_(err)_fqualmod & float & Jy & $500 \mu \mathrm{m}$ flux density (error) obtained from Herschel/SPIRE \\
\hline 47 & 112_AGN_afSta15_log & float & $\cdots$ & logarithmic decomposed $12 \mu \mathrm{m}$ AGN luminosity $\log \left(L_{12 \mu \mathrm{m}}^{(\mathrm{AGN})} / \mathrm{erg} \mathrm{s}^{-1}\right)$ \\
\hline 55 & CF_Sta16_tau9p7eq3_afSta15 & float & $\cdots$ & $C_{\mathrm{T}}($ dust $)$ \\
\hline 56 & SBtemplate_afSta15 & string & $\ldots$ & SB template used for the SED fitting this study: SB1-SB5 \\
\hline
\end{tabular}

Note. The detail of the selection of the flux is compiled in Section 2. The full catalog is available as a machine readable electronic table.

(This table is available in its entirety in machine-readable form.)

To acquire IR SEDs with a number of data points sufficient for spectral decomposition we require, for each source, at least three photometric bands within the rest-frame 3-500 $\mu \mathrm{m}$. This is because three data points are needed to define the normalization of the two components (AGN torus and host galaxy). Applying this criterion, our sample is reduced to 588 sources. In addition, we require at least one data point from either the NIR or FIR band to estimate the host galaxy component, which brings the sample to 587 sources. This is the final sample used for this study, and it represents a large fraction of the initial sample $(587 / 606=97 \%)$. The redshift distribution of the sample is shown in Figure $1 .^{21}$

\footnotetext{
${ }^{21}$ M81 is not shown in the figure due to its very low redshift of $z=10^{-4}$ (Ricci et al. 2017b).
}

We divide the sample into two AGN types based on $N_{\mathrm{H}}$ obtained by Ricci et al. (2017b). We define the AGNs with $N_{\mathrm{H}}<10^{22} \mathrm{~cm}^{-2}$ as unobscured AGNs, and those with $N_{\mathrm{H}} \geqslant 10^{22} \mathrm{~cm}^{-2}$ as obscured ones. Overall we have 300 unobscured and 287 obscured AGNs. The AGN types for the complete BAT 70 month catalog are tabulated in Ricci et al. (2017b), as well as in Table 1. We note that Koss et al. (2017) found a 95\% agreement for the unobscured and obscured AGNs with the presence of a broad $\mathrm{H} \beta$ line for optical types Seyfert 1-1.8 and Seyfert 2.

\section{Analysis}

We decompose the IR SEDs of AGNs using SB and AGN templates to estimate the intrinsic AGN IR luminosity. We use the IDL script DecompIR coded by Mullaney et al. (2011) and 


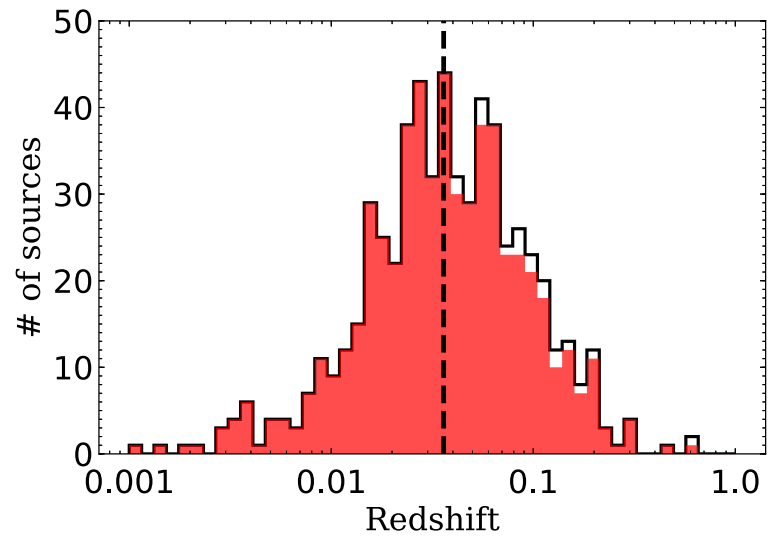

Figure 1. Redshift distribution of AGNs in the Swift/BAT 70 month catalog at Galactic latitude of $|b|>10^{\circ}$ (black solid line: 606 objects; see also Ichikawa et al. 2017) and of those used in this study (red area: 587 objects). The vertical dashed line represents the median redshift $(\langle z\rangle=0.037)$ in our sample.

further developed by Del Moro et al. (2013). This code accepts IR photometry points in the $3-500 \mu \mathrm{m}$ range as input and properly accounts for the filter and instrument response functions of the photometry points. It then computes the approximate levels of AGN and host galaxy contributions by fitting data that combine a host galaxy component with an AGN. DecompIR contains the mean AGN template produced from the Swift/BAT 9 month catalog (Tueller et al. 2008), which broadly traces the typical spectral forms of face-on and edge-on clumpy torus models (e.g., Nenkova et al. 2008a, 2008b) as shown in Mullaney et al. (2011). It also includes the five star-forming galaxy templates (Mullaney et al. 2011; Del Moro et al. 2013), using the average starburst SEDs derived by Dale et al. (2001). The five galaxy templates are composites of local star-forming galaxies with $L_{\mathrm{IR}}<10^{12} L_{\odot}$ (Brandl et al. 2006). They characterize well the full range of host galaxy SED shapes (Del Moro et al. 2013; Stanley et al. 2015), such as the galaxy template library of Chary \& Elbaz (2001). Using these representative templates, we are able to fit the data without suffering from the degeneracy of the fitting procedure caused by the large number of templates. In addition, some of our sources have only three data points, so it is reasonable to keep the number of free parameters as small as possible.

The free parameters of the fitting are the normalizations of the AGN and host galaxy templates; therefore at least three IR data points are needed to fit the SEDs. However, we added one more free parameter for only the very luminous sources. It is known that, in high-luminosity AGNs, the IR SEDs become much flatter at shorter wavelengths, which could be related to the stronger radiation field heating the surrounding dust to higher temperatures than in moderate-luminosity AGNs (e.g., Richards et al. 2006; Netzer et al. 2007; Mullaney et al. 2011; Symeonidis et al. 2016; Lani et al. 2017; Lyu et al. 2017). Our AGN SEDs also show such a tendency, especially at high luminosities $\left(L_{14-150}>10^{44} \mathrm{erg} \mathrm{s}^{-1}\right)$. Therefore, for the sources that have at least four data points and luminosities $L_{14-150}>10^{44} \mathrm{erg} \mathrm{s}^{-1}$, we also allow the spectral index $\alpha_{1}$ of the AGN template (see Mullaney et al. 2011) to be shallower at wavelengths shorter than $19 \mu \mathrm{m}$.

To determine the best fitting parameters, we first fit the SED by using the five host galaxy templates (SB1-SB5) and the AGN template. We then check the results obtained using the five different SB templates, and we choose the one that provides the best results according to the chi-squared statistic $\left(\chi^{2}\right)$ minimization.

Figure 2 shows examples of the best-fitting SEDs that include both the AGN and star formation components, together with the best-fitting SEDs that require only the host galaxy or the AGN component. All the other SEDs of our sample are compiled in the online journal. Overall, 474 sources required both the AGN and the host galaxy templates, while 94 sources required only the AGN template. For the latter objects, the fitting quality does not improve even when including an additional SB template. Since most of those sources (89 out of 94) are not detected in the FIR bands, and considering that the FIR bands have shallower sensitivities than the MIR ones, the lack of a significant contribution of the SB template in the MIR does not always imply that the host galaxy does not contribute to the total IR luminosity. In order to assess how much the host galaxy could contribute to the total infrared luminosity without affecting the observed SEDs, we calculate the upper limits on the contribution from star formation by following Stanley et al. (2015), where the same SED decomposition routine, DecompIR, was used. This was done by increasing the normalization of the host galaxy template until it reached one of the upper limits or exceeded the $3 \sigma$ uncertainty of a data point. We then used the starforming galaxy template that gave the highest value of IR luminosity as our conservative upper limit. For the sources that have an upper limit on the host galaxy component, we show the lower limits of the AGN contribution to the MIR flux (5-38 $\mu \mathrm{m}$; $\left.f_{\mathrm{AGN}}^{(\mathrm{MIR})}\right)$ and to the total IR flux $\left(5-1000 \mu \mathrm{m} ; f_{\mathrm{AGN}}^{(\mathrm{IR})}\right)$ in each SED, as illustrated in the middle panel of Figure 2. The lower limits on $f_{\mathrm{AGN}}^{(\mathrm{MIR})}$ and $f_{\mathrm{AGN}}^{(\mathrm{IR})}$ are reported in Table 1 , and readers can use the flag (flag_limit) to assess whether the values are lower limits or not.

There are 18 sources in our sample that were best fit to the host galaxy template alone $\left(f_{\mathrm{AGN}}^{(\mathrm{MIR})}=0\right)$. Again, in order to assess the contribution of AGNs to the total IR luminosity, we calculate the upper limits on the contribution from the AGN torus with the same methods as for the AGN-dominated SEDs, as discussed above. The upper limits of $f_{\mathrm{AGN}}^{(\mathrm{MIR})}$ and $f_{\mathrm{AGN}}^{(\mathrm{IR})}$ are also shown in the right panel of Figure 2 (see also Table 1).

Using this SED fitting approach, we have measurements of the AGN luminosity in the $12 \mu \mathrm{m}\left(L_{12 \mu \mathrm{m})}^{(\mathrm{AGN})}\right) \operatorname{MIR}\left(L_{\mathrm{MIR}}^{(\mathrm{AGN})}\right)$, and total IR $\left(L_{\mathrm{IR}}^{(\mathrm{AGN})}\right)$ bands. All the values, as well as the IR flux densities, are tabulated in Table 1. We do not compile the IR star-forming luminosity, due to the impossibility of obtaining reliable estimates for the sources not detected in the FIR.

\section{Results and Discussion}

\subsection{Fractional Luminosity Contribution of AGNs to the IR Band}

Figure 3 shows the median of the AGN contributions to the $12 \mu \mathrm{m}, \mathrm{MIR}$, and total IR luminosities as a function of $L_{14-150}$. The AGN contribution is calculated from the ratio of the AGN luminosity to the total (SF plus AGN) luminosity:

$$
\begin{aligned}
f_{\mathrm{AGN}}^{(12 \mu \mathrm{m}, \mathrm{MIR}, \mathrm{IR})}= & L_{12 \mu \mathrm{m}, \mathrm{MIR}, \mathrm{IR}}^{(\mathrm{AGN})} /\left(L_{12 \mu \mathrm{m}, \mathrm{MIR}, \mathrm{IR}}^{(\mathrm{AGN})}\right. \\
& \left.+L_{12 \mu \mathrm{m}, \mathrm{MIR}, \mathrm{IR}}^{(\mathrm{SF})}\right) .
\end{aligned}
$$

Figure 3 shows that the luminosity contribution of the AGNs to the $12 \mu \mathrm{m}, \mathrm{MIR}$, and total IR bands increases with $L_{14-195}$. At the low-luminosity end $\left(L_{14-195}<10^{43} \mathrm{erg} \mathrm{s}^{-1}\right)$, Figure 3 indicates that the host galaxy emission significantly 

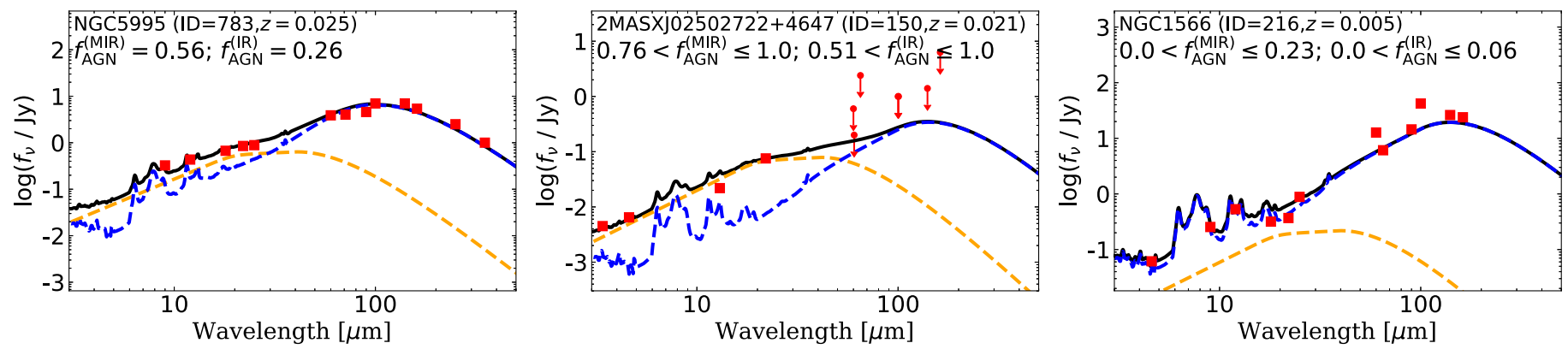

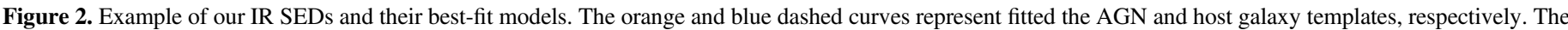

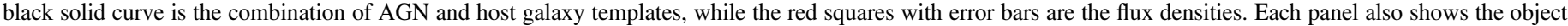

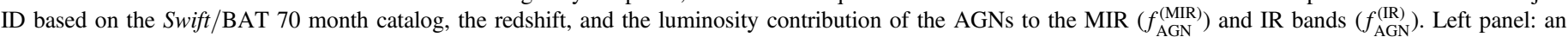

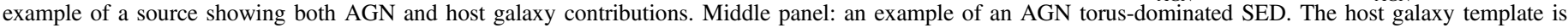

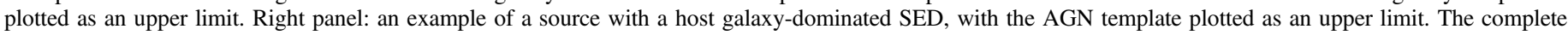
figure set of all SEDs of our sample (587 images) is available in the online journal.

(The complete figure set (587 images) is available.)

contaminates $(\simeq 50 \%-80 \%)$ the $12 \mu \mathrm{m}$ and MIR bands. At the high-luminosity end $\left(L_{14-195}>10^{43} \mathrm{erg} \mathrm{s}^{-1}\right)$, it clearly shows that the AGN component is the dominant $(\gtrsim 80 \%)$ energy source at $12 \mu \mathrm{m}$ and in the MIR band. This overall result is broadly consistent with previous studies that explored the AGN contribution using imaging with high spatial resolution (e.g., Asmus et al. 2011, 2014, and references therein). These works are discussed in Appendix A.1. On the other hand, in the total IR band, the AGN component contributes only up to $\simeq 50 \%$ even at high luminosities. This result is consistent with the calculations done for local quasars (Lyu et al. 2017), where it is shown that AGNs contribute $\simeq 50 \%$ of the flux even if they provide $90 \%$ of the MIR emission.

Figure 3 also shows that the scatter of the percentage is $\sim 20 \%$ for $f_{\mathrm{AGN}}^{(\mathrm{MIR})}$ and increases up to $\sim 35 \%$ for $f_{\mathrm{AGN}}^{\text {(IR) }}$. The scatter is mostly due to AGN-dominated sources without any detections in the FIR bands. Since no distant sources with $z>0.05$ have been observed with Herschel (see Meléndez et al. 2014; Shimizu et al. 2016), those sources have very shallow upper limits: $0.2 \mathrm{Jy}$ at $60 \mu \mathrm{m}$ (IRAS/FSC) and/or $0.55 \mathrm{Jy}$ at $90 \mu \mathrm{m}$ (AKARI/FIS). This allows a possible contribution of the host galaxy emission to the FIR bands, even when its contribution to the MIR flux is negligible as discussed in Section 3 (see also Lyu \& Rieke 2017). Therefore, FIR photometry with higher sensitivity is crucial to quantify the host galaxy contribution for those sources.

\subsection{IR Pure-AGN Candidates}

Some sources show AGN-dominated SEDs even in the FIR bands. These sources are called IR pure-AGN (Mullaney et al. 2011; Rosario et al. 2012, 2018; Matsuoka \& Woo 2015; Ichikawa et al. 2017), and they are ideal candidates for deriving intrinsic AGN IR templates. These IR pure-AGN have a spectral turnover at 20-40 $\mu \mathrm{m}$ (Alonso-Herrero et al. 2012; Hönig et al. 2014; Fuller et al. 2016; Lopez-Rodriguez et al. 2018) and a declining flux density from $40 \mu \mathrm{m}$ to $160 \mu \mathrm{m}$, suggesting a very low contribution from the starburst in the host galaxy. In order to check the SED turnover quantitatively, we plot IR color-color plots of $f_{70 \mu \mathrm{m}} / f_{160 \mu \mathrm{m}}$ versus $f_{22 \mu \mathrm{m}} / f_{70 \mu \mathrm{m}}$ in Figure 4. Both flux ratios are known to be sensitive to the SED peak, and therefore to the dust temperature (Meléndez et al. 2014; García-González et al. 2016b). The orange shaded area in Figure $4\left(f_{70 \mu \mathrm{m}} / f_{160 \mu \mathrm{m}}>1.0\right.$ and $\left.f_{22 \mu \mathrm{m}} / f_{70 \mu \mathrm{m}}>1.0\right)$ indicates a decline in flux density as a function of wavelength from $22 \mu \mathrm{m}$ to $160 \mu \mathrm{m}$ since the sources fulfill $f_{22 \mu \mathrm{m}}>$ $f_{70 \mu \mathrm{m}}>f_{160 \mu \mathrm{m}}$.

Figure 4 also shows the simulated IR color as a function of $f_{\mathrm{AGN}}^{(\mathrm{IR})}$ for the each SB template. All IR colors follow a similar trend: $f_{22 \mu \mathrm{m}} / f_{70 \mu \mathrm{m}}$ increases up to $f_{22 \mu \mathrm{m}} / f_{70 \mu \mathrm{m}} \simeq 1.0$ with $f_{\mathrm{AGN}}^{(\mathrm{IR})}$ up to 0.9 , while $f_{70 \mu \mathrm{m}} / f_{160 \mu \mathrm{m}}$ shows a very shallow increase until $f_{\mathrm{AGN}}^{(\mathrm{IR})} \leqslant 0.8$. However, for $f_{\mathrm{AGN}}^{(\mathrm{IR})}>0.9, f_{70 \mu \mathrm{m}} / f_{160 \mu \mathrm{m}}$ starts to drastically increase, reaching values up to $\simeq 7.0$. Thus, sources located in the orange shaded area should have AGNdominated IR SEDs with $f_{\mathrm{AGN}}^{(\mathrm{IR})}>0.90$. In this study we define a source as IR pure-AGN when it fulfills the following criteria: (1) $f_{\mathrm{AGN}}^{(\mathrm{IR})}>0.90$ and (2) a significant detection at both $60-70 \mu \mathrm{m}$ and $160 \mu \mathrm{m}$. A total of nine sources are selected with these criteria, and they are shown with the black crosses in Figure 4. Most IR pure-AGN are successfully located in the orange shaded area in the color-color plot. Figure 5 shows the SEDs of the nine selected IR pure-AGN. All sources show an SED turnover between $\simeq 20 \mu \mathrm{m}$ and $\simeq 70 \mu \mathrm{m}$, a declining flux density from $70 \mu \mathrm{m}$ to $160 \mu \mathrm{m}$, and no FIR bump due to star formation up to $90 \mu \mathrm{m}$, with the exception of Fairall 9 and II SZ 010. Some of the sources in our sample have already been reported as being dominated by emission from the torus in the IR from the study of their Spitzer/IRS spectra (e.g., MCG-0523-16; Ichikawa et al. 2015), based on the spectral turnover at 20-40 $\mu \mathrm{m}$ (Alonso-Herrero et al. 2012; Hönig et al. 2014; Fuller et al. 2016; Lopez-Rodriguez et al. 2018).

We also check the AGN properties of IR pure-AGN compared to the parent sample. The means and standard deviations of the logarithmic X-ray luminosity, black hole mass, and Eddington ratio of this subsample are $\left\langle\log L_{14-150}\right\rangle=43.7 \pm 0.3$ $\left\langle\log M_{\mathrm{BH}}\right\rangle=7.8 \pm 0.5$, and $\left\langle\log \lambda_{\text {Edd }}\right\rangle=-1.2 \pm 0.3$, respectively. These values are consistent with those of the parent sample of $\left\langle\log L_{14-150}\right\rangle=43.7 \pm 0.8,\left\langle\log M_{\mathrm{BH}}\right\rangle=8.0 \pm 0.8$, and $\left\langle\log \lambda_{\text {Edd }}\right\rangle=-1.5 \pm 0.8$. This result suggests that the dominant contribution of AGNs to the total IR band is not related to their higher AGN luminosities, lower BH masses, or higher Eddington ratio, while it implies that they have weaker star formation luminosities than other AGNs of similar luminosity. Actually, MCG-05-23-16 is one of the pure IRAGN whose $\mathrm{CO}$ emission has not been detected (Rosario et al. 2018) in the Swift/BAT AGN subset of the LLAMA survey (Davies et al. 2015). This suggests that its host galaxy already 

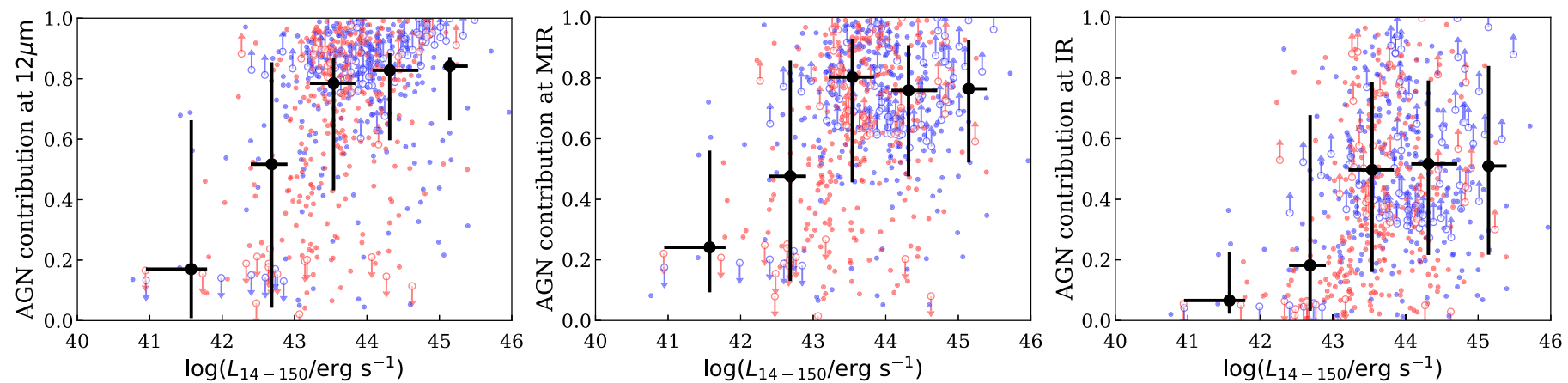

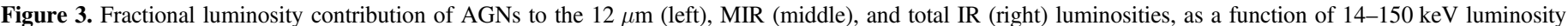

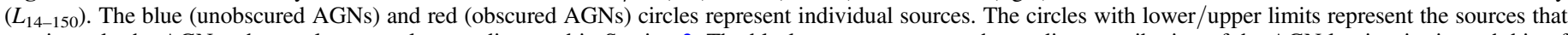

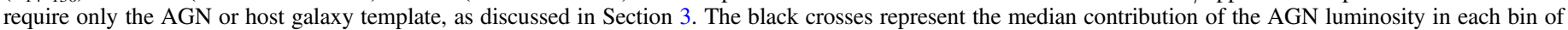
$L_{14-150}$, with the error bars showing the inter-percentage range containing $68.2 \%$ of the sample.

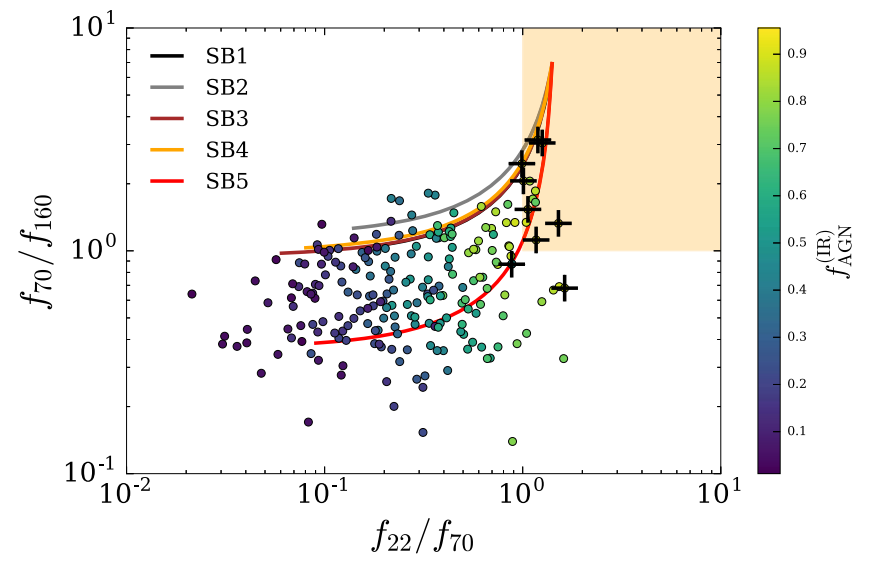

Figure 4. Observed ratio $f_{70 \mu \mathrm{m}} / f_{160 \mu \mathrm{m}}$ vs. $f_{22 \mu \mathrm{m}} / f_{70 \mu \mathrm{m}}$ for sample sources with secure detections in the $22 \mu \mathrm{m}, 70 \mu \mathrm{m}$, and $160 \mu \mathrm{m}$ bands. The color-color variations as a function of $f_{\mathrm{AGN}}^{(\mathrm{IR})}$ are also plotted for the five $\mathrm{SB}$ templates used in this study that originate from Mullaney et al. (2011). The black crosses are the IR pure-AGN sources discussed in Section 4.2. The color bar represents the AGN contribution to the total IR band $\left(f_{\mathrm{AGN}}^{(\mathrm{IR})}\right)$. The orange area illustrates the region with $f_{22 \mu \mathrm{m}}>f_{70 \mu \mathrm{m}}>f_{160 \mu \mathrm{m}}$.

lacks the molecular gas to produce the star formation. The ongoing molecular gas observations conducted by the BASS survey (M. Koss et al. 2018, in preparation) will explore the origin of the deficit of star formation in these IR pure-AGN sources.

\subsection{Correlation between the $12 \mu \mathrm{m}$ AGN and $14-150 \mathrm{keV}$ Luminosities}

Figure 6 shows the relation between $L_{12 \mu \mathrm{m})}^{(\mathrm{AGN})} L_{\mathrm{MIR}}^{(\mathrm{AGN})}$, and $L_{14-150}$ in the range $10^{40} \mathrm{erg} \mathrm{s}^{-1}<L_{14-150}<10^{47} \mathrm{erg} \mathrm{s}^{-1}$. Blue and red crosses represent unobscured and obscured AGNs, respectively. The upper limits, shown as open circles, represent the host galaxy-dominated sources that have a possible AGN contribution in the $12 \mu \mathrm{m}$ and MIR bands as discussed in Section 3. The slope of the relation between $L_{12 \mu \mathrm{m}}^{(\mathrm{AGN})}, L_{\mathrm{MIR}}^{(\mathrm{AGN})}$, and $L_{14-150}$ is estimated considering the two variables as independent parameters. Since our data contain both detections and upper limits, we apply the survival analysis method using the Python package $^{22}$ ASURV (Feigelson \& Nelson 1985; Isobe et al. 1986; Lavalley et al. 1992) to account for the upper limits on $L_{12 \mu \mathrm{m}}^{(\mathrm{AGN})}$ and $L_{\mathrm{MIR}}^{(\mathrm{AGN})}$. We use the slope bisector fits, which

\footnotetext{
${ }^{22}$ http://python-asurv.sourceforge.net/
}

minimize the perpendicular distance from the slope line to data points. The fits, with the form of $\log \left(L_{12 \mu \mathrm{m}, \mathrm{MIR}}^{(\mathrm{AGN})} / 10^{43} \mathrm{erg} \mathrm{s}^{-1}\right)=$ $(a \pm \Delta a)+(b \pm \Delta b) \log \left(L_{14-150} / 10^{43} \mathrm{erg} \mathrm{s}^{-1}\right)$, where $\Delta a$ and $\Delta b$ are the standard deviations of $a$ and $b$, respectively, result in

$$
\begin{aligned}
\log \frac{L_{12 \mu \mathrm{m}}^{(\mathrm{AGN})}}{10^{43} \mathrm{erg} \mathrm{s}^{-1}}= & (-0.24 \pm 0.03)+(1.08 \pm 0.03) \\
& \times \log \frac{L_{14-150}}{10^{43} \mathrm{erg} \mathrm{s}^{-1}}, \\
\log \frac{L_{\mathrm{MIR}}^{(\mathrm{AGN})}}{10^{43} \mathrm{erg} \mathrm{s}^{-1}}= & (-0.05 \pm 0.03)+(1.06 \pm 0.03) \\
& \times \log \frac{L_{14-150}}{10^{43} \mathrm{erg} \mathrm{s}^{-1}},
\end{aligned}
$$

and they are also summarized in Table 2. We find that both luminosity-luminosity and flux-flux correlations are significant (see also Appendix B for the flux-flux correlations).

In Figure 6, some of the fits reported by recent works are also overplotted. Since most previous studies used the 2-10 keV luminosity, we apply a conversion factor of $L_{14-150} / L_{2-10}=2.36$ under the assumption of the photon index $\Gamma=1.8$, which is the median value of the Swift/BAT 70 month AGN sample (Ricci et al. 2017b), for overplotting in the same figure. Since the AGN template used in this study has a ratio of $L_{\mathrm{MIR}}^{(\mathrm{AGN})} / L_{12 \mu \mathrm{m}}^{(\mathrm{AGN})}=1.92$, we also apply it to the slopes from the previous studies for overplotting in the relation between $L_{\mathrm{MIR}}^{(\mathrm{AGN})}$ and $L_{14-150}$.

Compared to Ichikawa et al. (2017), where we found $b=0.96 \pm 0.02$, the sample used here shows a smaller $12 \mu \mathrm{m}$ contribution from AGN at the low-luminosity end. This is because the sources with lower $L_{14-195}$ have a significant host galaxy contamination even in the MIR, as shown in Figure 3 and also in the right panel of Figure 11. Indeed, Ichikawa et al. (2017) also reported that the slope becomes slightly steeper with $b=1.05 \pm 0.03$ when one considers sources with $L_{14-195}>10^{43} \mathrm{erg} \mathrm{s}^{-1}$, for which the host galaxy contamination in the MIR is negligible. This is also consistent with the value of $b=1.08 \pm 0.03$ in this study.

We compare our results with what was found by Gandhi et al. (2009) and Asmus et al. (2015) using observations with high spatial resolution of X-ray-selected AGNs down to the low-luminosity end. The MIR emission in those studies is most likely dominated by the AGN torus, and they have a relatively 

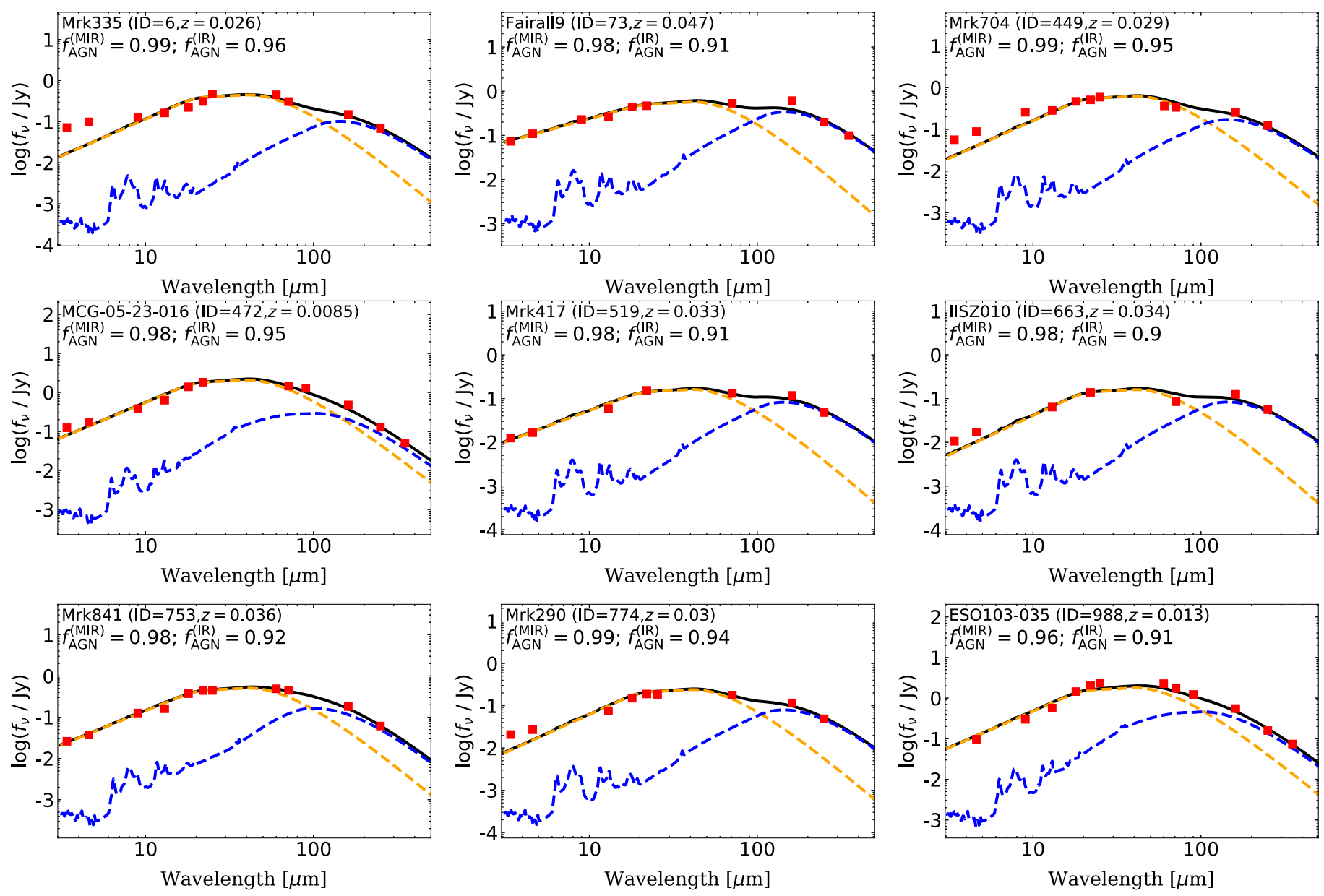

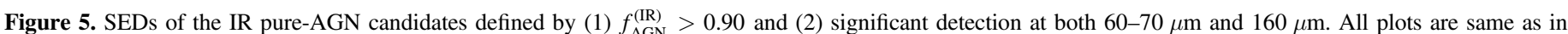
Figure 2.

low level of host galaxy contamination thanks to their spatially resolved images. As shown in Figure 6, our study finds a similar slope to that reported in Gandhi et al. (2009) ( $b=1.11 \pm 0.07)$, and it is also within the $3 \sigma$ uncertainty of that of Asmus et al. (2015) $(b=0.97 \pm 0.03)$. This strongly supports the idea that our SED decomposition method nicely reproduces the flux at high spatial resolution, which is thought to be dominated by AGN torus emission.

\subsection{Covering Factor of AGNs as a Function of Bolometric Luminosity}

The ratio of the AGN IR luminosity and the AGN bolometric luminosity $\left(R=L_{\mathrm{IR}}^{(\mathrm{AGN})} / L_{\mathrm{bol}}^{(\mathrm{AGN})}\right)$ has been interpreted as an indirect indicator of the dust covering factor $\left(C_{\mathrm{T}}\right.$ (dust)), since, for a given AGN luminosity, $L_{\mathrm{IR}}^{(\mathrm{AGN})}$ should be proportional to $C_{\mathrm{T}}$ (dust) $\left(L_{\mathrm{IR}}^{(\mathrm{AGN})} \propto C_{\mathrm{T}}\right.$ (dust) $\times L_{\text {bol }}^{(\mathrm{AGN})}$; Maiolino et al. 2007; Treister et al. 2008; Elitzur 2012). Since the flux of the accretion disk cannot be directly measured for all the sources of our sample, we used $L_{14-150}$ to estimate the bolometric luminosity. We apply a constant bolometric correction of $L_{\mathrm{bol}}^{(\mathrm{AGN})} / L_{2-10}=20$, which is equivalent to $L_{\mathrm{bol}}^{(\mathrm{AGN})} / L_{14-150}=8.47$ under the assumption of $\Gamma=1.8$, which is the median value of the Swift/BAT 70 month AGN sample (Ricci et al. 2017b). We note that our main results do not change significantly when adopting different bolometric corrections, including luminosity-dependent ones (Marconi et al. 2004). We briefly discuss this in Appendix C.2.

To calculate $R$, we proceed in the same manner as Stalevski et al. (2016). We use the total IR AGN luminosity integrated over $1-1000 \mu \mathrm{m}\left(L_{\mathrm{IR}}^{(\mathrm{AGN} ; 1-1000 \mu \mathrm{m})}\right)$ instead of $L_{\mathrm{IR}}^{(\mathrm{AGN})}$, which integrates the SED over 5-1000 $\mu \mathrm{m}$. This is because Stalevski et al. (2016) recommend using the AGN SEDs including NIR, which sometimes contributes to the total IR luminosity at a non-negligible level. Since we do not have an IR AGN template down to $1 \mu \mathrm{m}$, we extrapolate the AGN template using the same spectral index of $\alpha_{1}$ used at wavelengths shorter than $19 \mu \mathrm{m}$. Therefore, $R$ is calculated based on $R=$ $L_{\mathrm{IR}}^{(\mathrm{AGN} ; 1-1000 \mu \mathrm{m})} / L_{\mathrm{bol}}^{(\mathrm{AGN})}$ in the following study. Figure 7 shows the relation between $R$ and the AGN bolometric luminosity. The black dashed line represents the fit obtained using ASURV to account for the sources with an upper limit:

$$
\log R=(4.52 \pm 1.25)+(-0.12 \pm 0.03) \log \left(\frac{L_{\mathrm{bol}}^{(\mathrm{AGN})}}{\mathrm{erg} \mathrm{s}^{-1}}\right)
$$

This shows that $R$ is a very weak function of AGN bolometric luminosity. However, $R$ does not always represent the actual $C_{\mathrm{T}}$ (dust), because the standard geometrically thin and optically thick disk emits radiation anisotropically (Netzer 1987; Lusso et al. 2013). Thus we also estimate $C_{\mathrm{T}}$ (dust) exploiting the recent results of Stalevski et al. (2016), 

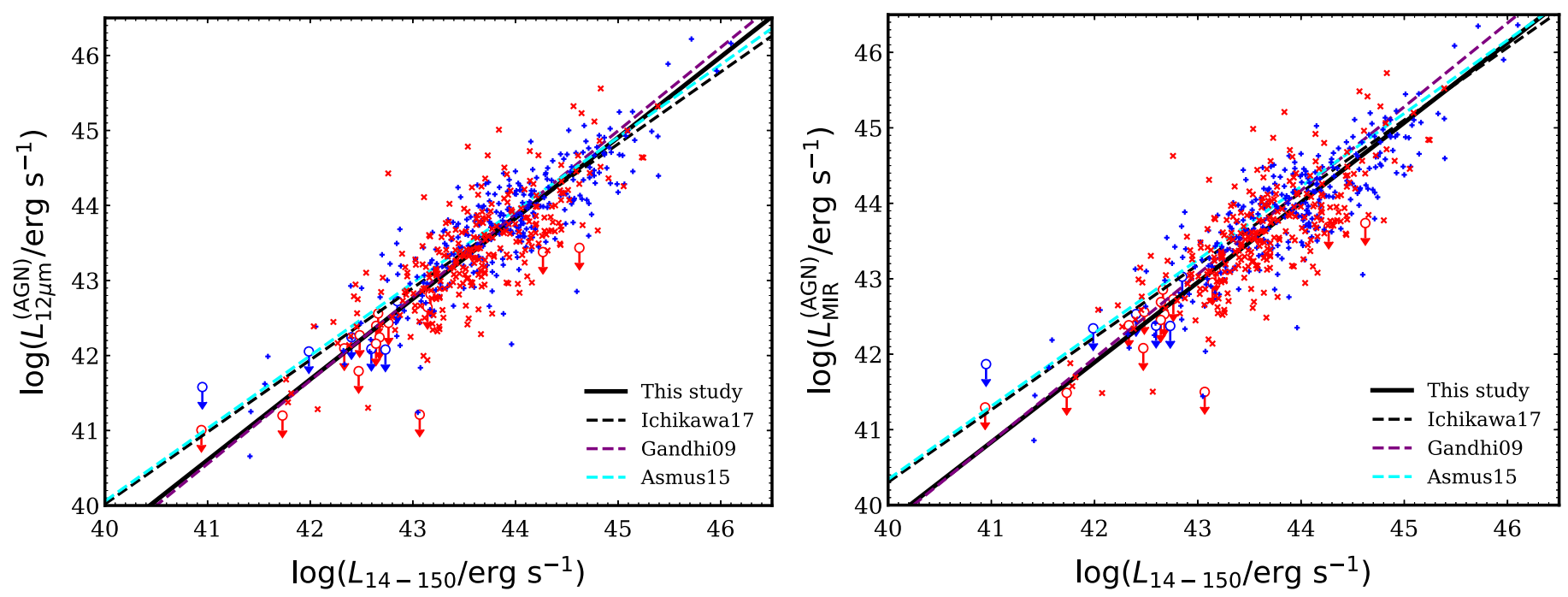

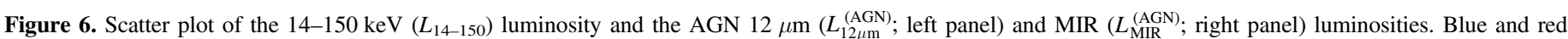

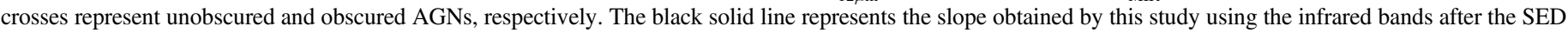

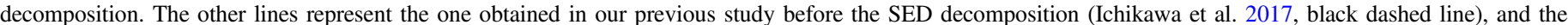
studies with higher spatial resolution by Gandhi et al. (2009, purple) and Asmus et al. (2015, cyan).

who computed the correction function between the covering factor $\left(C_{\mathrm{T}}\right.$ (dust)) and $R$ using a clumpy two-phase medium with a sharp boundary between the dusty and dust-free environments. They compute the $C_{\mathrm{T}}$ (dust) $-R$ relation for a range of equatorial torus thickness $\left(\tau_{9.7}=3-10\right)$. We consider here the function for $\tau_{9.7}=3$ :

$G_{T}($ dust $)=\left\{\begin{array}{c}-0.178 R^{4}+0.875 R^{3}-1.487 R^{2} \\ +1.408 R+0.192(\text { type } 1) \\ 2.039 R^{3}-3.976 R^{2}+2.765 R+0.205(\text { type } 2) .\end{array}\right.$

We use Equation (5) for type-1/type-2 AGN to unobscured/ obscured AGNs in this study. According to Stalevski et al. (2016) the relations reported above are valid only for $R \leqslant R_{\max }$, where $R_{\max }=1.3$ for unobscured $\mathrm{AGNs}$ and $R_{\max }=1.0$ for obscured AGNs, so we removed five sources with $R \geqslant R_{\max }$ from the sample. Figure 8 shows $C_{\mathrm{T}}$ (dust) as a function of $L_{\mathrm{bol}}$.

Besides the dust covering factor $C_{\mathrm{T}}$ (dust), we also calculate the fraction of obscured AGNs $\left(\log \left(N_{\mathrm{H}} / \mathrm{cm}^{-2}\right) \geqslant 22.0\right)$, including the Compton-thick sources for each $L_{\mathrm{bol}}^{(\mathrm{AGN})}$ bin as shown in Figure 8 (orange crosses). Since X-rays are absorbed by both gas and dust, the fraction of obscured AGNs is a proxy for the covering factor of the obscuring material, and is sensitive to both gas and dust $\left[C_{\mathrm{T}}\right.$ (gas + dust $\left.)\right] .{ }^{23}$ We follow the same approach to obtain $C_{\mathrm{T}}$ (gas + dust) as done by Ricci et al. (2017a). The column density $N_{\mathrm{H}}$ for our sample is obtained through detailed X-ray spectral fitting using follow-up X-ray observations (Ricci et al. 2017b). In the X-ray fitting, both photoelectric absorption and Compton scattering are considered, and they are listed in Table 5 of Ricci et al. (2017b). $C_{\mathrm{T}}$ (gas + dust) is defined as $C_{\mathrm{T}}($ gas + dust $)=f_{\text {Cthin }}+f_{\mathrm{CT}}$, where $f_{\mathrm{Cthin}}$ is the fraction of

\footnotetext{
23 Although the dusty region also contains the gas, in this study we use $C_{\mathrm{T}}$ (dust) as the covering area of dust which is heated by AGN, and re-emits the IR. We then use $C_{\mathrm{T}}$ (gas + dust) as the covering area of gas that is responsible for the X-ray absorption. This region includes (1) the dusty region defined by $C_{\mathrm{T}}$ (dust) since that region also includes the gas, and (2) the dust-free region that is inside the sublimation radius but contains the neutral gas.
}

Compton-thin obscured AGNs $\left(22 \leqslant \log \left(N_{\mathrm{H}} / \mathrm{cm}^{-2}\right)<24.0\right)$ in each $L_{\text {bol }}^{(\mathrm{AGN})}$ bin, while the Compton-thick fraction is $f_{\mathrm{CT}}=0.32$ for $\log \left(L_{\mathrm{bol}}^{(\mathrm{AGN})} / \mathrm{erg} \mathrm{s}^{-1}\right)<43.5$ and $f_{\mathrm{CT}}=0.21$ for $\log \left(L_{\text {bol }}^{(\mathrm{AGN})} / \mathrm{erg} \mathrm{s}^{-1}\right)>43.5$ obtained from the intrinsic $N_{\mathrm{H}}$ distribution (Ricci et al. 2015). The reason for using $f_{\mathrm{CT}}$ above is because even though Swift/BAT sources are unbiased for $N_{\mathrm{H}}<10^{24} \mathrm{~cm}^{-2}$, they can still be affected by obscuration for $N_{\mathrm{H}}>10^{24} \mathrm{~cm}^{-2}$.

$$
\text { 4.4.1. } L_{\mathrm{bol}}^{(\mathrm{AGN})} \text {-dependent Trend of } C_{\mathrm{T}} \text { (dust) }
$$

Figure 8 shows that both $C_{\mathrm{T}}$ (dust) and $C_{\mathrm{T}}$ (gas + dust) seem to decrease as functions of AGN bolometric luminosity, and at the high-luminosity end the two finally converge. This luminosity-dependent trend of $C_{\mathrm{T}}$ has been observationally reported in multiple wavelengths from studies in the IR (e.g., Maiolino et al. 2007; Alonso-Herrero et al. 2011), optical (Simpson 2005), and X-rays (Ueda et al. 2003, 2011, 2014; Beckmann et al. 2009; Ricci et al. 2013).

However, recent studies have also reported contradictory results that the luminosity dependence of $C_{\mathrm{T}}$ (dust) is actually really weak, or that the trend even disappears after considering some possible biases. Netzer et al. (2016) argue that using the different bolometric corrections would make the reported luminosity dependence of $C_{\mathrm{T}}$ (dust) disappear. Stalevski et al. (2016) also found that the dependence on luminosity is always less pronounced after considering the anisotropy of the emission from the torus. A similar weak or insignificant dependence on luminosity is reported by Mateos et al. (2016), and a more detailed review is given by Netzer (2015).

In order to understand this trend in more detail, we conduct a simulation to assess the luminosity dependence of $C_{\mathrm{T}}$ (dust). We first generate the two random populations of $L_{14-150}$ for unobscured and obscured AGNs in a total of $10^{4}$ samples with the same number ratio as our parent sample (unobscured/ obscured $=300 / 287$; see Section 2). Each sample is generated based on our parent sample, using a Gaussian distribution with median $\log \left(L_{14-150} / \mathrm{erg} \mathrm{s}^{-1}\right)$ of $(43.9,43.6)$ and standard deviation of ( $0.85 \mathrm{dex}, 0.67 \mathrm{dex})$ for unobscured and obscured AGNs, respectively. Then the distribution of $L_{\mathrm{IR}}^{(\mathrm{AGN} ; 1-1000 \mu \mathrm{m})}$ is 
Table 2

Equations of the Correlation in This Study

\begin{tabular}{lcccc}
\hline \hline 1$)$ & $(2)$ & $(3)$ & $(4)$ & $(5)$ \\
$Y$ & $X$ & $a$ & $b$ & Reference \\
\hline $\log \left(\frac{L_{12 \mu \mathrm{m}}^{(\mathrm{AGN})}}{10^{43} \mathrm{erg} \mathrm{s}^{-1}}\right)$ & $\log \left(\frac{L_{14-150}}{10^{43} \mathrm{erg} \mathrm{s}^{-1}}\right)$ & $-0.24 \pm 0.03$ & $1.08 \pm 0.03$ & Section 4.3 \\
$\log \left(\frac{L_{\mathrm{MR}}^{(\mathrm{AGN})}}{10^{43} \mathrm{erg} \mathrm{s}^{-1}}\right)$ & $\log \left(\frac{L_{14-150}}{10^{43} \mathrm{erg} \mathrm{s}^{-1}}\right)$ & $-0.05 \pm 0.03$ & $1.06 \pm 0.03$ & Section 4.3 \\
$\log R$ & $\log \left(\frac{L_{\text {bol }}^{(\mathrm{AGN})}}{\operatorname{erg~s}^{-1}}\right)$ & $4.52 \pm 1.25$ & $-0.12 \pm 0.03$ & Section 4.4
\end{tabular}

Note. Correlation properties between two physical values. Columns: (1) $Y$ variable; (2) $X$ variable; (3) regression intercept (a) and its $1 \sigma$ uncertainty; (4) slope (b) and its $1 \sigma$ uncertainty; the equation is represented as $Y=a+b X$; (5) reference for the details of each equation.

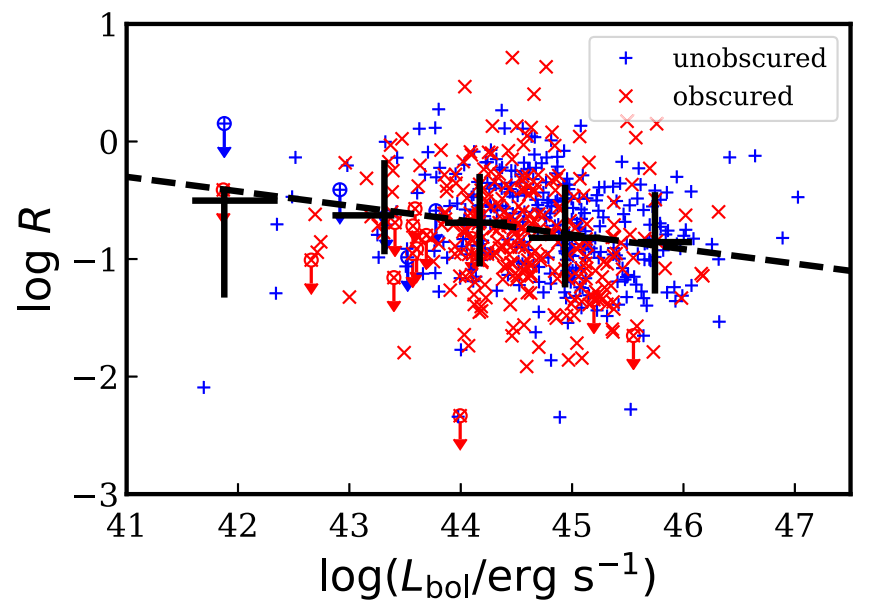

Figure 7. $R=L_{\mathrm{IR}}^{(\mathrm{AGN} ; 1-1000 \mu \mathrm{m})} / L_{\mathrm{bol}}^{(\mathrm{AGN})}$ as a function of the bolometric luminosity. The black crosses represent the median value of $R$ in each bin of bolometric luminosity, with the error bars showing the inter-percentage range containing $68.2 \%$ of the sample.

calculated under the assumption that two populations follow the luminosity correlation of $L_{\mathrm{IR}}^{(\mathrm{AGN} ; 1-1000 \mu \mathrm{m})}-L_{14-150}$ with a scatter of $\sigma=0.4$ dex, and finally the distribution of $C_{\mathrm{T}}$ (dust) is computed in the same manner. The result is shown in Figure 9: the computed $C_{\mathrm{T}}$ (dust) distribution (gray cross bins) roughly reproduces the luminosity dependence of the black solid bins. Next, we assume that all AGNs should follow the luminosity correlation of $L_{\mathrm{IR}}^{(\mathrm{AGN} ; 1-1000 \mu \mathrm{m})}-L_{14-150}$ and the intrinsic population should have the narrower scatter, down to $\sigma=0.1$ dex. The result is plotted with pink bins in Figure 9, showing that the luminosity dependence has disappeared and the binned $C_{\mathrm{T}}$ (dust) has an almost constant value of $C_{\mathrm{T}}(\mathrm{dust}) \simeq 0.4$ over the entire $L_{\mathrm{bol}}^{(\mathrm{AGN})}$ range. Therefore, we conclude that this apparent dependence on luminosity can be produced purely by the scatter of the distribution, and our results confirm the recent arguments that the luminosity dependence of $C_{\mathrm{T}}$ (dust) is actually really weak, or that the trend even disappears.

\subsubsection{Relation between $C_{\mathrm{T}}$ (dust) and $C_{\mathrm{T}}$ (gas + dust)}

The other interesting result from Figure 8 is that $C_{\mathrm{T}}$ (gas + dust) is always same as or larger than the binned $C_{\mathrm{T}}$ (dust) over the entire AGN luminosity range. This relation still holds if $C_{\mathrm{T}}$ (dust) $\simeq 0.4 \leqslant C_{\mathrm{T}}$ (gas + dust) in our simulation as shown in Figure 9. This result suggests the presence of dust-free gas, possibly located in the broad-line region (BLR), and is responsible for part of the X-ray absorption. Observationally, using long-term X-ray data, Markowitz et al. (2014) found evidence of occultation events in the X-rays, and the locations of those gas clumps are in the dust-free region or at the inner edge of the dusty torus (e.g., Risaliti et al. 2007, 2011; Maiolino et al. 2010; Ricci et al. 2016). In addition, Minezaki \& Matsushita (2015) and Gandhi et al. (2015) have suggested that the location of narrow $\mathrm{Fe} \mathrm{K} \alpha$ line-emitting material could be between the BLR and the dusty torus. Those observations imply the presence of gas at radii inside the sublimation radius. Several studies have also proposed that the AGN gas disk inside the dust sublimation radius could significantly contribute to the observed column density in Compton-thick AGNs, since such disks are often found to have large inclination angles (e.g., Davies et al. 2015; Masini et al. 2016; Ramos Almeida \& Ricci 2017). We also check whether the similar trend of $C_{\mathrm{T}}$ (dust) $\leqslant C_{\mathrm{T}}$ (gas + dust) can be seen using only the MIR fluxes before the SED decomposition. This is discussed in Appendix C.1.

Figure 8 also shows that both $C_{\mathrm{T}}$ (dust) and $C_{\mathrm{T}}$ (gas + dust) seem to suggest a peak at $\log L_{\text {bol }} \simeq 43$, and they both seem to decrease at lower luminosities. However, since the number of samples is limited in this bin range, we cannot confirm the statistical significance of this trend at the current stage (see also the discussion in Appendix C.2).

\subsubsection{Comparison of $C_{\mathrm{T}}$ (dust) between Unobscured and Obscured AGNs}

We compare $C_{\mathrm{T}}$ (dust) between the AGN subgroups. The left panel of Figure 10 shows $C_{\mathrm{T}}$ (dust) of unobscured (blue) and obscured (red) AGNs as a function of $L_{\text {bol }}^{(\mathrm{AGN})}$. Although the scatter is large, the binned $C_{\mathrm{T}}$ (dust) of obscured AGNs is always systematically higher than that of unobscured AGNs.

The right panel of Figure 10 shows the distribution of $C_{\mathrm{T}}$ (dust) for unobscured (blue) and obscured (red) AGNs. The $C_{\mathrm{T}}$ (dust) distribution for unobscured AGNs is clustered at smaller values of $\left\langle C_{\mathrm{T}}\right.$ (dust) $\rangle=0.41$, while obscured AGNs are distributed over a wider $C_{\mathrm{T}}$ (dust) range, reaching $C_{\mathrm{T}}($ dust $) \simeq$ 1.0. We apply the Kolmogorov-Smirnov (KS) test to these two samples: the $p$-value of the null hypothesis is $5.7 \times 10^{-8}$ and the KS statistic is 0.24 , suggesting that the two distributions are 


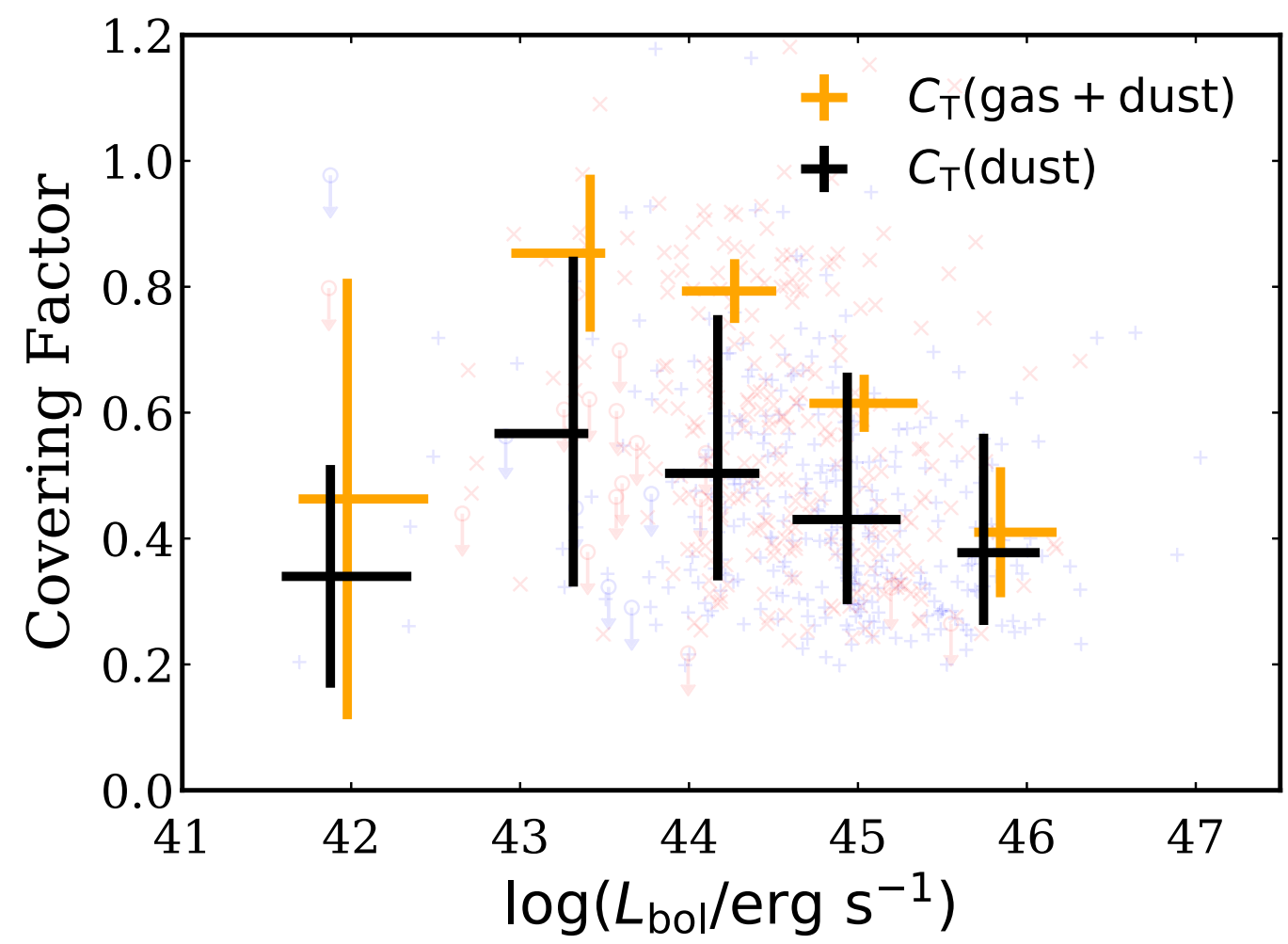

Figure 8. The covering factor $\left(C_{\mathrm{T}}\right)$ as a function of the bolometric luminosity. The dust covering factors $C_{\mathrm{T}}($ dust $)$ are obtained from $R$ using the corrections reported in Stalevski et al. (2016). The covering factors of gas and dust $C_{\mathrm{T}}$ (gas+dust) are obtained from the X-ray observations and the spectral fitting based on the obscured AGN fraction including Compton-thick AGNs (Ricci et al. 2015, 2017b). The Compton-thick fraction is $f_{\mathrm{CT}}=0.32$ for $\log \left(L_{\mathrm{bol}}^{(\mathrm{AGN})} / \mathrm{erg} \mathrm{s}^{-1}\right)<43.5$ and $f_{\mathrm{CT}}=0.21$ for $\log \left(L_{\mathrm{bol}}^{(\mathrm{AGN})} / \mathrm{erg} \mathrm{s}^{-1}\right)>43.5$. The orange crosses are shifted to the right by 0.1 dex for clarity.

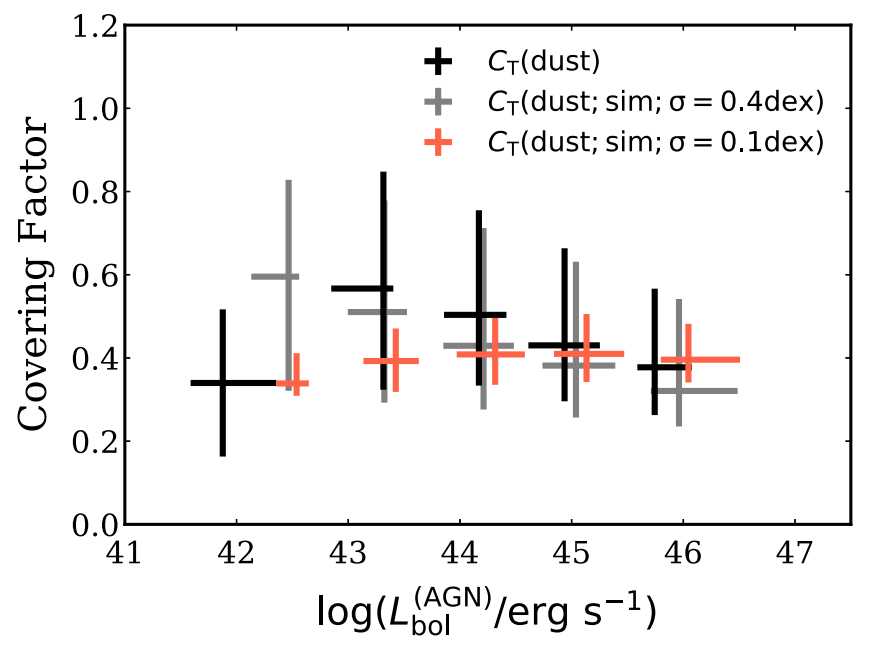

Figure 9. The dust covering factor $\left(C_{\mathrm{T}}(\mathrm{dust})\right)$ as a function of the bolometric luminosity. The simulated dust covering factor $C_{\mathrm{T}}$ (dust; sim) is obtained from the simulation using a random population following the $L_{\mathrm{IR}}^{(\mathrm{AGN})}-L_{14-150}$ relation with a scatter of $\sigma=0.4 \mathrm{dex}$ (gray crosses) and $\sigma=0.1 \mathrm{dex}$ (pink crosses). The gray/pink crosses are shifted to the right by $0.1 / 0.2$ dex for clarity.

significantly different, assuming that the significance level is $\alpha=0.05$.

One possible origin of the difference is that the smaller $C_{\mathrm{T}}$ (dust) for unobscured AGNs could be due to larger $L_{\mathrm{bol}}^{(\mathrm{AGN})}$. However, as discussed in Section 4.4.1, the luminosity dependence of $C_{\mathrm{T}}$ (dust) is unlikely, and the KS test shows that the distribution of $C_{\mathrm{T}}$ (dust) for unobscured and obscured AGNs is statistically significant even in each $L_{\text {bol }}^{(\mathrm{AGN})}$ bin for $42.5<$ $\log \left(L_{\mathrm{bol}}^{(\mathrm{AGN})} / \mathrm{erg} \mathrm{s}^{-1}\right)<47$, with $p$-values of $p<10^{-5}$ for $42.5<\log \left(L_{\mathrm{bol}}^{(\mathrm{AGN})} / \mathrm{erg} \mathrm{s}^{-1}\right)<45.5$ and $p=0.02$ for $45.5<$ $\log \left(L_{\text {bol }}^{(\mathrm{AGN})} / \mathrm{erg} \mathrm{s}^{-1}\right)<47$.

Another possible interpretation of the difference is as a consequence of the selection of unobscured and obscured AGNs. Several authors argue that AGN classification depends on the distribution of $C_{\mathrm{T}}$ (dust); unobscured AGNs would be preferentially observed from AGNs with lower $C_{\mathrm{T}}$ (dust), and obscured AGNs from AGNs with higher $C_{\mathrm{T}}$ (dust) (e.g., Ramos Almeida et al. 2011; Elitzur 2012; Ichikawa et al. 2015; Lanz et al. 2018).

\section{Conclusions}

We have constructed the IR (3-500 $\mu \mathrm{m})$ SED for 587 nearby AGNs detected in the 70 month Swift/BAT all-sky survey. Using this almost complete (587 out of 606; 94\%) sample, we have decomposed the IR $(3-500 \mu \mathrm{m})$ SEDs into SB and AGN components. The decomposition enabled us to estimate the AGN contribution to the $12 \mu \mathrm{m}\left(L_{12 \mu \mathrm{m}}^{(\mathrm{AGN})}\right)$, MIR $\left(L_{\mathrm{MIR}}^{(\mathrm{AGN})}\right)$, and total IR $\left(L_{\mathrm{IR}}^{(\mathrm{AGN})}\right)$ luminosities, as well as the contribution of AGN luminosity to the $12 \mu \mathrm{m}\left(f_{\mathrm{AGN}}^{(12 \mu \mathrm{m})}\right)$, MIR $\left(f_{\mathrm{AGN}}^{(\mathrm{MIR})}\right)$, and total $\mathrm{IR}\left(f_{\mathrm{AGN}}^{\mathrm{IR})}\right)$ emission. Our results are summarized as follows.

1. The luminosity contribution of the AGN to the $12 \mu \mathrm{m}$, MIR, and total IR band flux increases with the $14-150 \mathrm{keV}$ luminosity. The AGN contributions to the $12 \mu \mathrm{m}, \mathrm{MIR}$, and total IR are almost $80 \%, 80 \%$, and $50 \%$ at the high-luminosity end, respectively. 

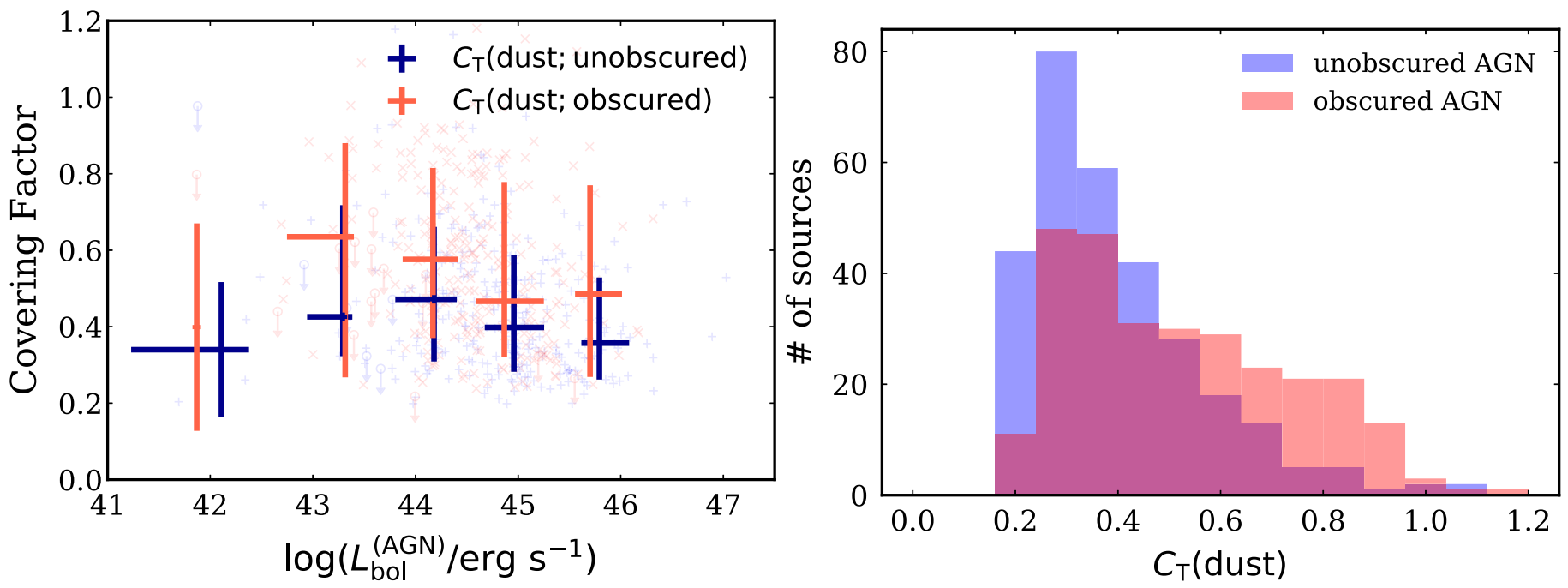

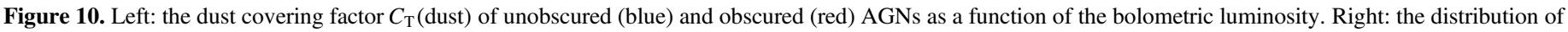
$C_{\mathrm{T}}$ (dust) of unobscured and obscured AGNs.

2. We find nine pure IR-AGN whose IR emission is dominated by the AGN torus at least up to $90 \mu \mathrm{m}$. These pure IR-AGN could be good candidates to create templates of the IR AGN SED, with an expanded range up to $90 \mu \mathrm{m}$. Those sources could be easily selected using the color selection of $f_{70 \mu \mathrm{m}} / f_{160 \mu \mathrm{m}}>1.0$ and $f_{22 \mu \mathrm{m}} / f_{70 \mu \mathrm{m}}>1.0$.

3. We find a good luminosity correlation between the MIR and ultrahard X-ray bands over five orders of magnitude $\left(41<\log \left(L_{14-150} / \mathrm{erg} \mathrm{s}^{-1}\right)<46\right)$. Our slope is almost consistent with that obtained by studies carried out using observations with high spatial resolution of nearby Seyfert galaxies, supporting our SED decomposition method, which would nicely estimate the intrinsic MIR emission without the contamination of star formation from the host galaxies.

4. We find that the average of the covering factor of gas and dust inferred from X-ray observations always exceeds the average of the covering factor of the dust torus, suggesting that the dust-free gas contributes to the absorption in X-rays. This gas could be located inside the dust sublimation radius, in agreement with previous observations based on X-ray occultation and spectral fitting studies of nearby AGNs.

5. The luminosity-dependent trend of $C_{\mathrm{T}}$ (dust) might originate from the large scatter of the luminosity correlations between $L_{\mathrm{IR}}^{(\mathrm{AGN} ; 1-1000 \mu \mathrm{m})}$ and $L_{14-150}$, and the trend would disappear once the scatter is removed.

6. Obscured AGNs tend to have larger $C_{\mathrm{T}}$ (dust) than unobscured AGNs. This difference originates from the AGN classification, which depends on the distribution of the obscuring material.

We thank the anonymous referee for a careful reading of the manuscript and helpful suggestions that greatly strengthened the paper. We thank James Mullaney and Agnese Del Moro for providing the SB SED templates in this study, and Satoshi Takeshige for the technical discussion of IDL routine. We also thank Masatoshi Imanishi, Ryo Tazaki, and Daniel Asmus for fruitful discussions. K.I. thanks the Department of Astronomy at Kyoto university, where a part of the research was conducted. This study benefited from financial support from the Grant-in-Aid for JSPS fellow for young researchers (P.D., K.I.), JSPS KAKENHI (18K13584; K.I.), and JST grant "Building of Consortia for the Development of Human Resources in Science and Technology" (K.I.). C.R. acknowledges the CONICYT+PAI Convocatoria Nacional subvencion a instalacion en la academia convocatoria año 2017 PAI77170080. F.E.B. acknowledges support from CONICYT-Chile (Basal-CATA PFB-06/2007, FONDECYT Regular 1141218), the Ministry of Economy, Development, and Tourism's Millennium Science Initiative through grant IC120009, awarded to The Millennium Institute of Astrophysics, MAS. K.O. is an International Research Fellow of the Japan Society for the Promotion of Science (JSPS) (ID: P17321). D.J.R. acknowledges the support of UK Science and Technology Facilities Council through grant code ST/ P000541/1.

\section{Appendix A Comparison with Studies from the Literature \\ A.1. Comparison with the High-spatial-resolution Flux Obtained with Ground-based $8 \mathrm{~m}$ Class Telescopes}

Here we compare the results in this study with the highspatial-resolution observations by Asmus et al. (2014, 2015). Out of 122 high-spatial-resolution sources, we found 112 sources also used in this study. The remaining 10 sources were not found because they are located at low Galactic latitudes $|b|<10^{\circ}$, which we initially removed from the parent sample as discussed in Ichikawa et al. (2017).

The top left panel of Figure 11 shows the $12 \mu \mathrm{m}$ luminosity correlation between the high-spatial-resolution MIR observations $\left(L_{12 \mu \mathrm{m}}^{\text {(Asmus) }}\right.$; Asmus et al. 2014, 2015) and this study after the SED decomposition $\left(L_{12 \mu \mathrm{m}}^{(\mathrm{AGN})}\right)$. The figure clearly shows that our decomposition method successfully follows the one-to-one relation with the high-spatial-resolution observations down to 

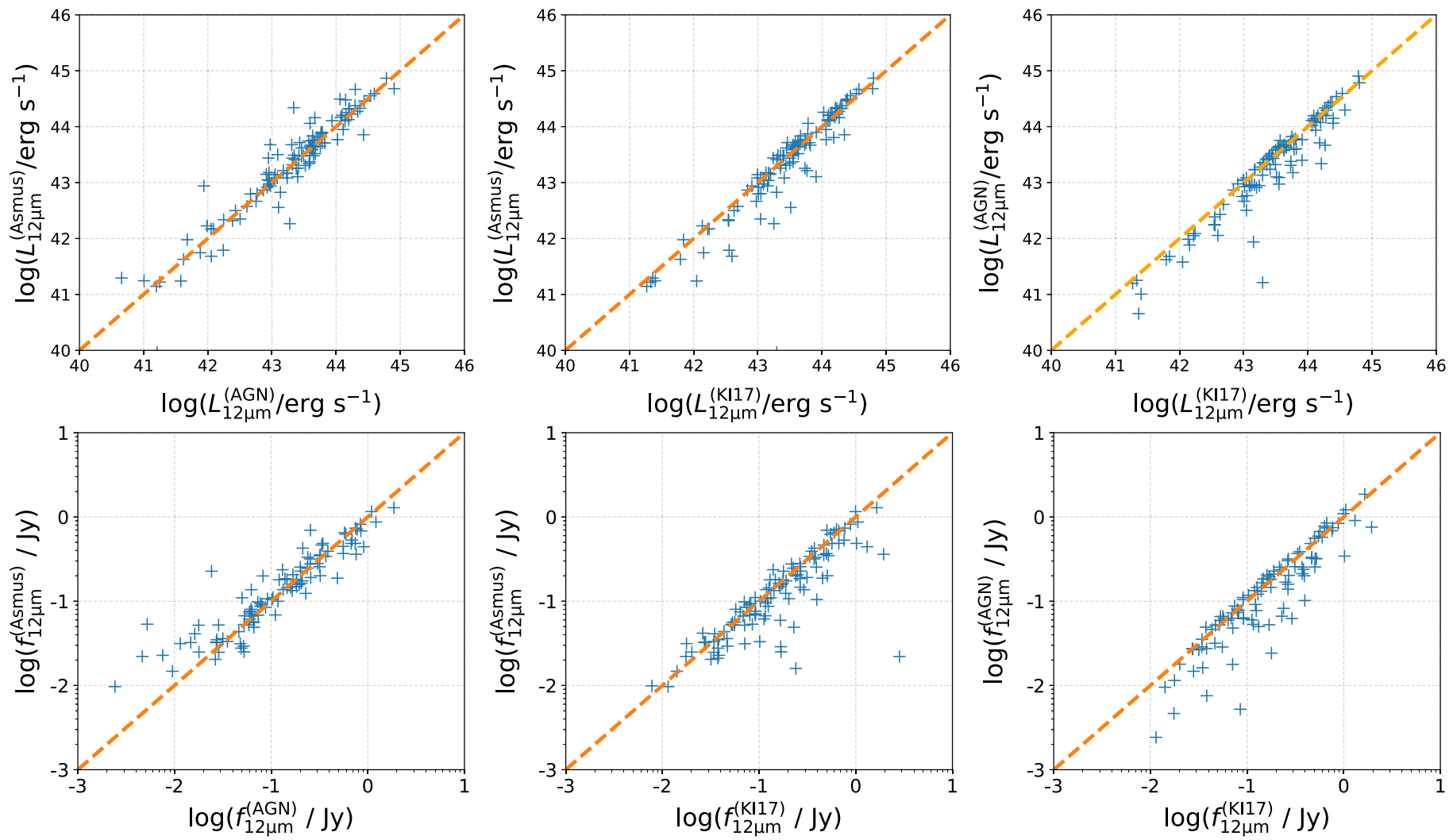

Figure 11. Top: scatter plot of the $12 \mu \mathrm{m}$ luminosities obtained from high-spatial-resolution MIR observations ( $L_{12 \mu \mathrm{m}}^{\text {(Ass); }}$; Asmus et al. 2014, 2015), this study after the SED decomposition $\left(L_{12 \mu \mathrm{m})}^{(\mathrm{AGN})}\right)$ and before the SED decomposition $\left(L_{12 \mu \mathrm{m}}^{(\mathrm{KII})}\right.$; Ichikawa et al. 2017). Blue crosses represent individual sources, and the orange dashed line represents the 1:1 relation. Panel show the luminosity relations between $L_{12 \mu \mathrm{m}}^{(\text {(Asmus) }}$ and $L_{12 \mu \mathrm{m}}^{(\mathrm{AGN})}$ (left), $L_{12 \mu \mathrm{m}}^{(\mathrm{Asmus})}$ and $L_{12 \mu \mathrm{m}}^{(\mathrm{KII7})}$ (middle), $L_{12 \mu \mathrm{m}}^{(\mathrm{AGN})}$ and $L_{12 \mu \mathrm{m}}^{(\mathrm{KII7})}$ (right). Bottom: same plots as top but for $12 \mu \mathrm{m}$ flux densities.

$\log \left(L_{12 \mu \mathrm{m}}^{(\mathrm{AGN})} / \mathrm{erg} \mathrm{s}^{-1}\right) \simeq 41.0$. The average of two parameters is $\left\langle\log L_{12 \mu \mathrm{m}}^{\text {(Asmus) }} / L_{12 \mu \mathrm{m}}^{(\mathrm{AGN})}\right\rangle=0.05$. The standard deviation is $\sigma=0.36$

The top middle and top right panels of Figure 11 show the luminosity relation between $L_{12 \mu \mathrm{m}}^{\text {(Asmus) }}, L_{12 \mu \mathrm{m}}^{(\mathrm{AGN})}$, and the lowresolution $12 \mu \mathrm{m}$ luminosity before the SED decomposition $\left(L_{12 \mu \mathrm{m}}^{(\mathrm{KI})}\right)$, which is taken from Ichikawa et al. (2017). Both panels show that the points are distributed equally to or below the one-to-one relations and suggest contamination of the host galaxy component in $L_{12 \mu \mathrm{m}}^{(\mathrm{KI} 17)}$. The mean and standard deviation $\operatorname{are}\left\langle\log L_{12 \mu \mathrm{m}}^{\text {(Asmus) }} / L_{12 \mu \mathrm{m}}^{(\mathrm{KIIT})}\right\rangle=-0.10 \pm 0.43$. This shows that the correlation between $L_{12 \mu \mathrm{m}}^{(\mathrm{Asmus})}$ and $L_{12 \mu \mathrm{m}}^{(\mathrm{AGN})}$ is tighter than that between $L_{12 \mu \mathrm{m}}^{(\text {(Asmus) }}$ and $L_{12 \mu \mathrm{m}}^{(\mathrm{KI})}$, indicating that our decomposition method nicely reduces the contamination in the $12 \mu \mathrm{m}$ band from the host galaxies.

The bottom panels of Figure 11 show the same relations as those in the top panels, but for $12 \mu \mathrm{m}$ flux densities. All three panels also show a similar trend to the luminosity relations. One notable difference is that the flux density of the highspatial-resolution observation $\left(f_{12 \mu \mathrm{m}}^{\text {(Asmus) }}\right)$ shows a decline in the number of sources at around $f_{12 \mu \mathrm{m}}^{\text {(Asmus) }} \simeq 10^{-2} \mathrm{Jy}$. This is almost consistent with the lower bound of the flux density observable with ground-based $8 \mathrm{~m}$ class telescopes with significant signalto-noise ratio (Asmus et al. 2014). Our study can explore flux densities down to $10^{-3} \mathrm{Jy}$, which is equivalent to the detection limit of the WISE W3 $(12 \mu \mathrm{m})$ band. This is one of the advantages of the SED decomposition method using lowresolution, but sensitive space IR satellites compared to ground-based studies.

\section{A.2. Comparison with Different Models from the Literature}

In this appendix we briefly compare the IR AGN luminosity obtained in this study and the ones obtained in Shimizu et al. (2017). They applied a different IR SED model to the IR data set, which is similar to ours but obtained from the Herschel observations in the Swift/BAT 58 month AGN catalog to study mainly the global star-forming properties in the host galaxies. Instead of using the AGN/host galaxy templates, they provided functions of the hot dust and the host galaxy respectively by following Casey (2012), and their functions are given by

$$
f(\nu)=N_{\mathrm{pl}}\left(\frac{\nu}{\nu_{\mathrm{c}}}\right) e^{-\left(\nu_{\mathrm{c}} / \nu\right)^{2}}+S_{\mathrm{MBB}}\left(\nu, M_{\text {dust }}, T_{\text {dust }}\right),
$$

where the first term stands for the AGN component with the normalization $N_{\mathrm{pl}}$ and cut-off frequency $\nu_{\mathrm{c}}$, and the second term represents the host galaxy component of a single modified blackbody with a parameter of dust mass $M_{\text {dust }}$ and a dust temperature $T_{\text {dust }}$. The fitting method used in their study is also different from ours. They use a Bayesian framework with a 

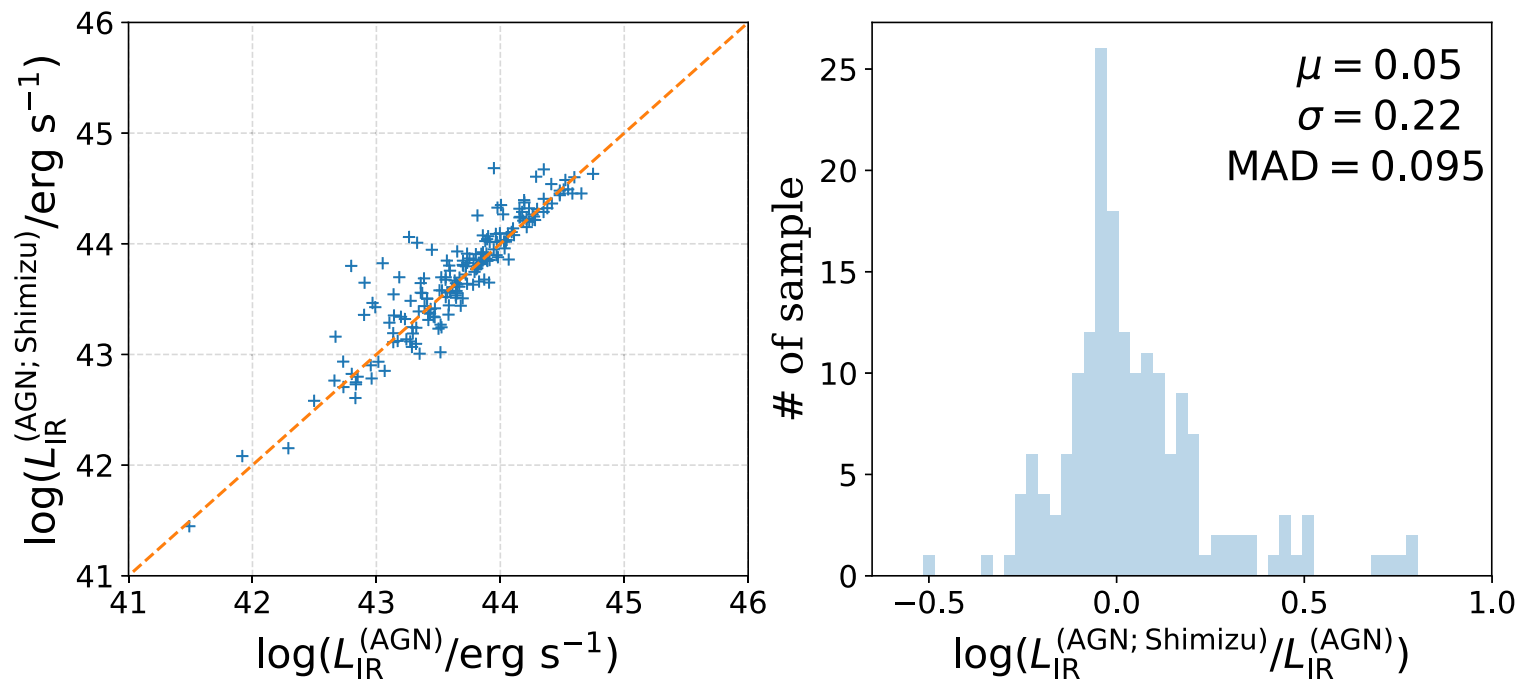

Figure 12. Left: scatter plot of total IR AGN luminosities obtained from Shimizu et al. (2017) $\left(L_{\mathrm{IR}}^{(\mathrm{AGN} ; \text { Shimizu })}\right)$ and those obtained from this study $\left(L_{\mathrm{IR}}^{(\mathrm{AGN})}\right)$. Blue crosses represent individual sources, and the orange dashed line represents the $1: 1$ relation. Right: histogram of $r=\log \left(L_{\mathrm{IR}}^{(\mathrm{AGN} ; \text { Shimizu })} / L_{\mathrm{IR}}^{(\mathrm{AGN})}\right)$. The mean $\mu$, standard deviation $\sigma$, and median absolute deviation (MAD) of $r$ are also shown.

Markov chain Monte Carlo procedure to obtain the posterior probability distribution function, and then use the median to obtain the best fitted parameters. Out of 307 sources in their sample, 204 sources have at least one Herschel detection and a reliable fitting quality ( $I$ ir_agn_flag $=0$ ). After crossmatching with our sample, we found 180 sources in common. Again, the 24 sources removed are located at low Galactic latitude $|b|<10^{\circ}$.

Since Shimizu et al. (2017) do not provide any $12 \mu \mathrm{m}$ AGN flux or luminosity, we compare the total IR AGN luminosity obtained from their AGN component. The left panel of Figure 12 shows the correlation between the IR AGN luminosities obtained from Shimizu et al. (2017) $\left(L_{\mathrm{IR}}^{\text {(AGN;Shimizu) }}\right)$ and the ones from this study. We find a good luminosity correlation between $L_{\mathrm{IR}}^{(\mathrm{AGN} ; \text { Shimizu) }}$ and $L_{\mathrm{IR}}^{(\mathrm{AGN})}$. The Spearman's rank coefficient is 0.91 , and probability of the null hypothesis is $P=4.9 \times 10^{-69}$, suggesting that the correlation is significant. The average of the distribution of $r=$ $\log \left(L_{\mathrm{IR}}^{(\mathrm{AGN} ; \mathrm{Shimizu})} / L_{\mathrm{IR}}^{(\mathrm{AGN})}\right)$ is also shown in the right panel of Figure 12. We do not find any systematic offset between the two methods $(\mu=0.05)$ with a standard deviation of $\sigma=0.22$ dex. Since there are several outliers with $\log \left(L_{\mathrm{IR}}^{(\mathrm{AGN} ; \text { Shimizu })} / L_{\mathrm{IR}}^{(\mathrm{AGN})}\right)>0.3$, we also compute the median absolute deviation (MAD) and the value is MAD $=0.095$ dex, which is smaller than the standard deviation by a factor of two. As already mentioned in Shimizu et al. (2017), their model allows the power-law component to extend to longer wavelengths, which would return slightly larger AGN luminosities with $\log \left(L_{\mathrm{IR}}^{(\mathrm{AGN} ; \text { Shimizu })} / L_{\mathrm{IR}}^{(\mathrm{AGN})}\right)>0.3$ for some cases. Those sources are actually seen in Figure 12 but they are only a small percentage of the sample. Thus, we conclude that, although the fitting methods and the template are different, each model returns the consensus results for the estimation of the IR AGN luminosities.

\section{Appendix B \\ Flux Correlation between $12 \mu \mathrm{m}, \mathrm{MIR}$, and 14-150 keV Bands}

Figure 13 shows the flux correlation between the AGN $12 \mu \mathrm{m}$, MIR, and $14-150 \mathrm{keV}$ bands, revealing a clear correlation between the bands even in the flux-flux plane. The Spearman's rank coefficient is 0.43 and the probability of the null hypothetical is $P=10^{-28}$ for both flux-flux correlations, suggesting that the correlation is significant. The slopes are $b=1.48$ for the AGN $12 \mu \mathrm{m}$ band and $b=1.49$ for the AGN MIR band, respectively. As we discussed in Ichikawa et al. (2017), there is a clear decline in the number of sources at $f_{14-150}<10^{-11} \mathrm{erg} \mathrm{s}^{-1} \mathrm{~cm}^{-2}$, while MIR flux can go down to $3 \times 10^{-13} \mathrm{erg} \mathrm{s}^{-1} \mathrm{~cm}^{-2}$, which is the typical detection limit of the MIR band. This trend suggests that the sample is limited by the detection limit of the X-ray flux. 

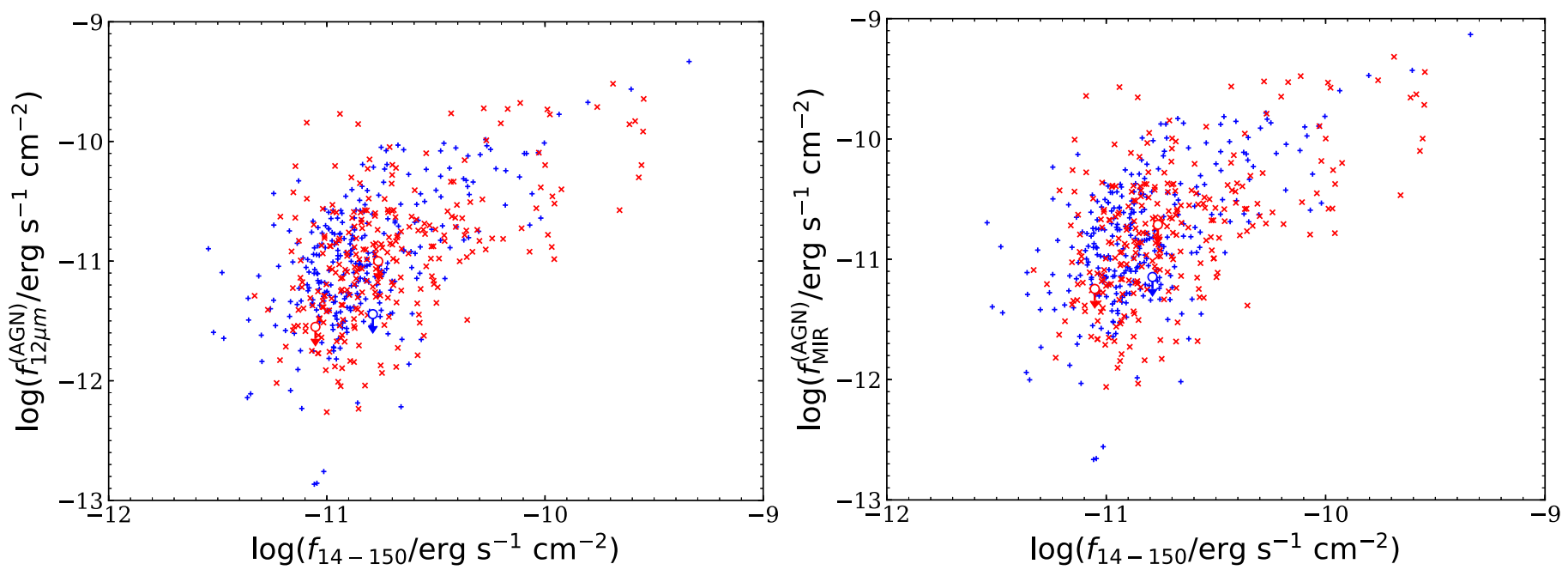

Figure 13. Correlation between the fluxes in the $12 \mu \mathrm{m}$, MIR, and 14-150 keV bands. Blue and red crosses represent unobscured and obscured AGNs, respectively.

\section{Appendix C \\ Comparison of Relation between $C_{\mathrm{T}}$ and $L_{\mathrm{bol}}^{(\mathrm{AGN})}$ using Different Values}

\section{C.1. $C_{\mathrm{T}}$ (dust) Estimated from the Observed $12 \mu \mathrm{m}$ Luminosity}

It is important to check whether the same result in Figure 8 is obtained using the MIR fluxes without host galaxy subtraction. To achieve this, we estimate the total IR AGN luminosity by assuming that the observed $12 \mu \mathrm{m}$ luminosity originates from the AGN emission. Then we use the conversion factor of $L_{\mathrm{IR}}^{(\mathrm{AGN} ; 1-1000 \mu \mathrm{m})} / L_{12 \mu \mathrm{m}}^{(\mathrm{AGN})}=2.77$ estimated from the AGN template in this study. The calculation of $R$ and then $C_{\mathrm{T}}$ (dust) is performed in the same manner as we discussed in Section 4.4. The left panel of Figure 14 shows the relation between $C_{\mathrm{T}}$ and $L_{\mathrm{bol}}^{(\mathrm{AGN})}$ using $C_{\mathrm{T}}$ (dust) estimated above. It clearly shows that while the result $C_{\mathrm{T}}$ (dust) $<C_{\mathrm{T}}$ (gas + dust) holds for $43.5<\log L_{\mathrm{bol}}^{(\mathrm{AGN})}<45.5, C_{\mathrm{T}}$ (dust) becomes almost equal to $C_{\mathrm{T}}$ (gas + dust) in the luminosity bin $42.5<$ $\log L_{\text {bol }}^{(\mathrm{AGN})}<43.5$, which is not seen in Figure 8. We also apply the KS test between $C_{\mathrm{T}}$ (dust) and $C_{\mathrm{T}}$ (gas + dust) for each $L_{\text {bol }}^{(\mathrm{AGN})}$ luminosity bin. In order to apply this test, we make a Gaussian distribution of $C_{\mathrm{T}}$ (gas + dust) in which the central value is the average of $C_{\mathrm{T}}$ (gas + dust) and $1 \sigma$ is its standard deviation, and the number of sources is the same as for $C_{\mathrm{T}}$ (dust) in the same $L_{\mathrm{bol}}^{(\mathrm{AGN})}$ bin. As a result, we find a significant difference for the luminosity bins with $43.5<\log L_{\text {bol }}^{(\mathrm{AGN})}<45.5$ with $p$-values of $p<10^{-30}$, while the clear significance is not obtained for the luminosity bins $\log L_{\mathrm{bol}}^{(\mathrm{AGN})}<43.5 \quad(p>0.5)$ and $45.5<\log L_{\mathrm{bol}}^{(\mathrm{AGN})}(p=$ $0.26)$. This difference originates from the flux subtraction after the SED decomposition, especially at the lower AGN luminosity end, suggesting its importance and its effect on estimating the dust covering factor.

\section{C.2. Dependence of the Bolometric Corrections}

Here we summarize whether different bolometric corrections can affect the relation shown in Figure 8. In this study, following the method used in Ricci et al. (2017a), we use a constant bolometric correction of $L_{\mathrm{bol}}^{(\mathrm{AGN})} / L_{14-150}=8.47$, which is based on $L_{\mathrm{bol}}^{(\mathrm{AGN})} / L_{2-10}=20$ under the assumption of $\Gamma=1.8$, the median value of the Swift/BAT 70 month AGN sample (Ricci et al. 2017b). On the other hand, Marconi et al. (2004) account for variations in AGN SEDs to obtain the bolometric correction with AGN luminosity. They assume a varying relation between optical/UV and X-ray luminosity, which is called a luminosity-dependent bolometric correction. This gives a larger bolometric correction than the constant one at the higher AGN luminosity end, which would make average $L_{\text {bol }}^{(\text {AGN) }}$ larger and $C_{\mathrm{T}}$ smaller.

The right panel of Figure 14 shows the same plot as Figure 8, but using the luminosity-dependent bolometric correction of Marconi et al. (2004). As expected from the luminosity-dependent bolometric correction, the distribution is slightly shifted to the right and downward in the figure. Actually, the median values of $\mathrm{AGN}$ bolometric luminosity and $C_{\mathrm{T}}$ (dust) change from $\left(\log L_{\mathrm{bol}}^{(\mathrm{AGN})}, C_{\mathrm{T}}\right.$ (dust) $)=(44.65,0.46)$ to $\left(\log L_{\mathrm{bol}}^{\text {(AGN;M04) }}\right.$, $C_{\mathrm{T}}($ dust $\left.)\right)=(44.79,0.39)$.

The figure clearly retains the trend of $C_{\mathrm{T}}$ (gas + dust $) \geqslant$ $C_{\mathrm{T}}$ (dust) over the entire AGN luminosity range. On the other hand, the slight decline in $C_{\mathrm{T}}$ (dust) in the lowest AGN bolometric luminosity bin disappears in Figure 14. This is mainly because of the small statistics in the lowest luminosity bin and some sources being shifted into a higher luminosity bin because of the larger bolometric correction by Marconi et al. (2004).

\section{C.3. Dependence of Additional Torus Parameters}

We here discuss how the dust covering factor changes when we change the set of the torus parameters. In this study we have only considered the spectral power-law index $\left(\alpha_{1}\right)$ at $\lambda<19 \mu \mathrm{m}$ for the high-luminosity end with $\log L_{14-150}>44$, and not considered the dust extinction for obscured AGNs, which could be one of the most significant parameters shaping the torus SEDs. The left panel of Figure 15 shows $C_{\mathrm{T}}$ (dust) as a function of $L_{\mathrm{bol}}^{(\mathrm{AGN})}$ after addition of the dust extinction for obscured AGNs using the absorption profile of Draine (2003) (see also Mullaney et al. 2011). $C_{\mathrm{T}}$ (dust) becomes slightly larger, but the overall sense does not change. The middle panel shows the same plot using a fixed power-law index $\alpha_{1}=1.8$ for all sources without the dust extinction. $C_{\mathrm{T}}$ (dust) shows a flatter distribution than Figure 8 , but the overall trend of $C_{\mathrm{T}}$ (dust) $<C_{\mathrm{T}}$ (gas + dust) still holds. 

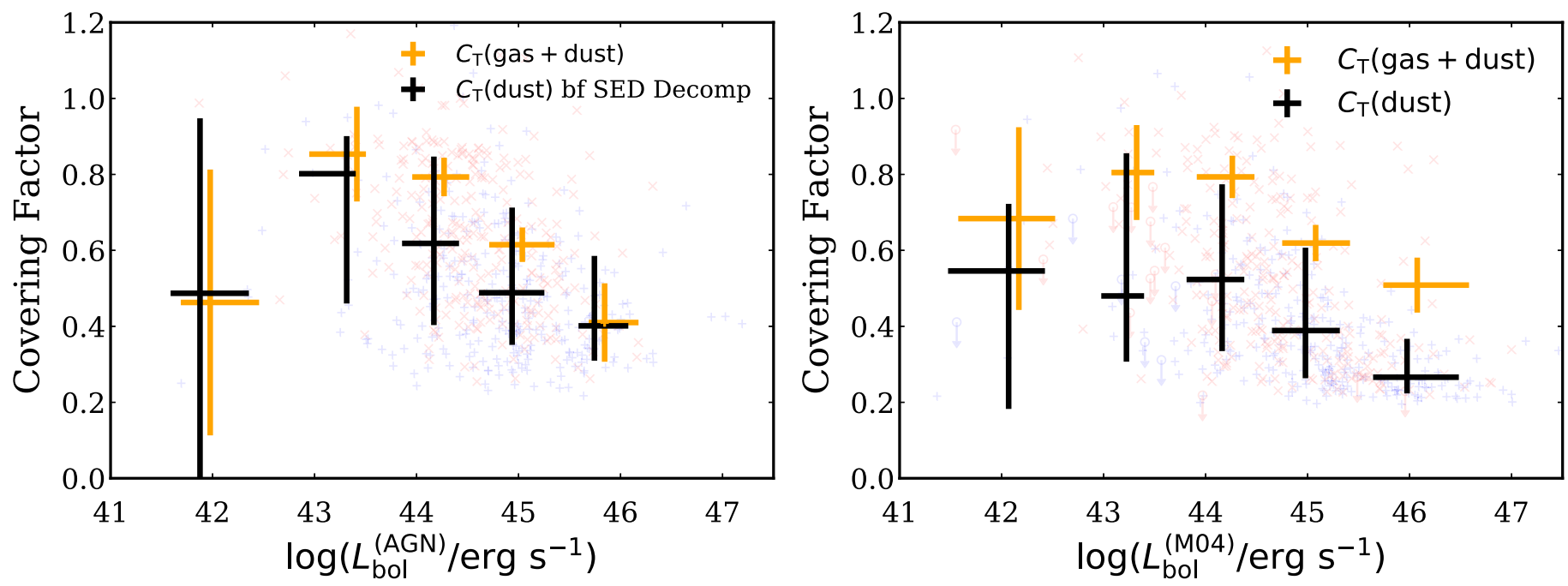

Figure 14. Same as Figure 8, but estimating the values differently. Left: the covering factors used here are based on the estimation using the observed $12 \mu \mathrm{m}$ luminosities in Ichikawa et al. (2017) before the IR SED decomposition. Right: the bolometric corrections used are dependent on the bolometric luminosity ( $L_{\text {bol }}^{\text {(M04); }}$ Marconi et al. 2004), not the constant bolometric correction.
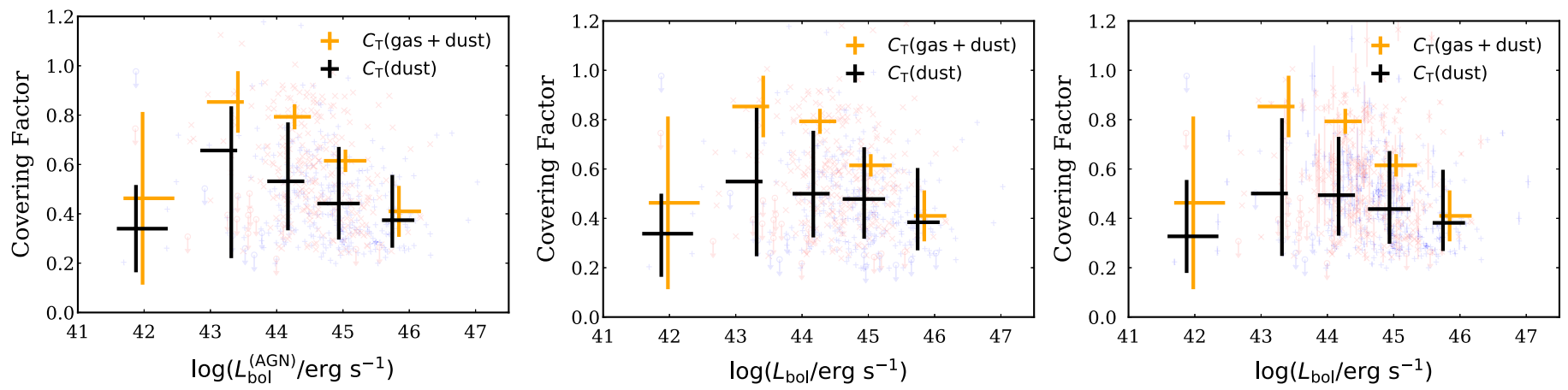

Figure 15. Same as Figure 8, but using a different set of free parameters for (left) the addition of dust extinction and (middle) a fixed power-law index $\alpha_{1}=1.8$. Right: same as Figure 8, but using the averaged $C_{\mathrm{T}}$ (dust) of the SB templates as discussed in Appendix C.4.

\section{C.4. Dependence of Other SB Templates}

In this study, we used the best SB template based on the lowest $\chi^{2}$ value as discussed in Section 3. However, other SB templates sometimes show fitting results of similar quality with small $\Delta \chi^{2}$ between the best one and the others. Therefore, we investigate here how the result could be affected by using such different SB templates. We consider here that the fitting result is indistinguishable if $\Delta \chi^{2}$ between the best fitting SB template and the other SB ones is smaller than $\chi_{\max }^{2}$, which is the maximum allowed $\chi^{2}$ corresponding to a $p$-value of $p=0.05$ of $\chi^{2}$ distributions with the degree of freedom for each source. If one source has $\geqslant 2$ indistinguishable SB templates, we then measure the averaged $C_{\mathrm{T}}$ (dust) and the standard deviation $\Delta C_{\mathrm{T}}$ (dust). The right panel of Figure 15 shows the result using the averaged $C_{\mathrm{T}}$ (dust) here, and the binned values of $C_{\mathrm{T}}$ (dust) become slightly smaller than the original ones.

\section{ORCID iDs}

Kohei Ichikawa (ib https://orcid.org/0000-0002-4377-903X Claudio Ricci $\odot$ https://orcid.org/0000-0001-5231-2645 Yoshihiro Ueda (1) https://orcid.org/0000-0001-7821-6715 Franz E. Bauer (i) https://orcid.org/0000-0002-8686-8737 Taiki Kawamuro (10) https://orcid.org/0000-0002-6808-2052 Michael J. Koss (1) https://orcid.org/0000-0002-7998-9581
Kyuseok Oh (1) https://orcid.org/0000-0002-5037-951X

David J. Rosario (1) https://orcid.org/0000-0002-0001-3587

T. Taro Shimizu (10 https://orcid.org/0000-0002-2125-4670

Marko Stalevski (10 https://orcid.org/0000-0001-5146-8330

Benny Trakhtenbrot (1) https://orcid.org/0000-0002-3683-7297

\section{References}

Alexander, D. M., \& Hickox, R. C. 2012, NewAR, 56, 93

Alonso-Herrero, A., Esquej, P., Roche, P. F., et al. 2016, MNRAS, 455, 563

Alonso-Herrero, A., Pereira-Santaella, M., Rieke, G. H., \& Rigopoulou, D. 2012, ApJ, 744, 2

Alonso-Herrero, A., Ramos Almeida, C., Esquej, P., et al. 2014, MNRAS, 443, 2766

Alonso-Herrero, A., Ramos Almeida, C., Mason, R., et al. 2011, ApJ, 736, 82

Asmus, D., Gandhi, P., Hönig, S. F., Smette, A., \& Duschl, W. J. 2015, MNRAS, 454, 766

Asmus, D., Gandhi, P., Smette, A., Hönig, S. F., \& Duschl, W. J. 2011, A\&A, 536, A36

Asmus, D., Hönig, S. F., \& Gandhi, P. 2016, ApJ, 822, 109

Asmus, D., Hönig, S. F., Gandhi, P., Smette, A., \& Duschl, W. J. 2014, MNRAS, 439, 1648

Baumgartner, W. H., Tueller, J., Markwardt, C. B., et al. 2013, ApJS, 207, 19 Beckmann, V., Soldi, S., Ricci, C., et al. 2009, A\&A, 505, 417

Beichman, C. A., Neugebauer, G., Habing, H. J., Clegg, P. E., \& Chester, T. J. 1988, Infrared Astronomical Satellite (IRAS) Catalogs and Atlases. Vol. 1: Explanatory Supplement

Brandl, B. R., Agócs, T., Aitink-Kroes, G., et al. 2016, Proc. SPIE, 9908, 990820 Brandl, B. R., Bernard-Salas, J., Spoon, H. W. W., et al. 2006, ApJ, 653, 1129 Burtscher, L., Hönig, S., Jaffe, W., et al. 2016, Proc. SPIE, 9907, 99070R 
Burtscher, L., Meisenheimer, K., Tristram, K. R. W., et al. 2013, A\&A, 558, A149 Casey, C. M. 2012, MNRAS, 425, 3094

Chary, R., \& Elbaz, D. 2001, ApJ, 556, 562

Colina, L., Gonzalez Delgado, R., Mas-Hesse, J. M., \& Leitherer, C. 2002, ApJ, 579, 545

Comastri, A., Gilli, R., Marconi, A., Risaliti, G., \& Salvati, M. 2015, A\&A, 574, L10

Cutri, R. M., Wright, E. L., Conrow, T., et al. 2013, in Explanatory Supplement to the AllWISE Data Release Products, ed. R. M. Cutri et al.

da Cunha, E., Charlot, S., \& Elbaz, D. 2008, MNRAS, 388, 1595

Dale, D. A., Helou, G., Contursi, A., Silbermann, N. A., \& Kolhatkar, S. 2001, ApJ, 549, 215

Davies, R. I., Burtscher, L., Rosario, D., et al. 2015, ApJ, 806, 127

Del Moro, A., Alexander, D. M., Mullaney, J. R., et al. 2013, A\&A, 549, A59

Delvecchio, I., Gruppioni, C., Pozzi, F., et al. 2014, MNRAS, 439, 2736

Draine, B. T. 2003, ARA\&A, 41, 241

Elitzur, M. 2012, ApJL, 747, L33

Esquej, P., Alonso-Herrero, A., González-Martín, O., et al. 2014, ApJ, 780, 86 Feigelson, E. D., \& Nelson, P. I. 1985, ApJ, 293, 192

Fuller, L., Lopez-Rodriguez, E., Packham, C., et al. 2016, MNRAS, 462, 2618

Gandhi, P., Hönig, S. F., \& Kishimoto, M. 2015, ApJ, 812, 113

Gandhi, P., Horst, H., Smette, A., et al. 2009, A\&A, 502, 457

García-González, J., Alonso-Herrero, A., Hernán-Caballero, A., et al. 2016a MNRAS, 458, 4512

García-Burillo, S., Combes, F., Ramos Almeida, C., et al. 2016, ApJL, 823, L12

García-González, J., Alonso-Herrero, A., Hernán-Caballero, A., et al. 2016b, MNRAS, 458, 4512

González-Martín, O., Masegosa, J., Hernán-Caballero, A., et al. 2017, ApJ, 841, 37

González-Martín, O., Rodríguez-Espinosa, J. M., Díaz-Santos, T., et al. 2013, A\&A, 553, A35

Griffin, M. J., Abergel, A., Abreu, A., et al. 2010, A\&A, 518, L3

Hatziminaoglou, E., Fritz, J., Franceschini, A., et al. 2008, MNRAS, 386, 1252

Hernán-Caballero, A., Alonso-Herrero, A., Hatziminaoglou, E., et al. 2015 ApJ, 803, 109

Hönig, S. F., Gandhi, P., Asmus, D., et al. 2014, MNRAS, 438, 647

Hönig, S. F., Kishimoto, M., Antonucci, R., et al. 2012, ApJ, 755, 149

Hönig, S. F., Kishimoto, M., Gandhi, P., et al. 2010, A\&A, 515, A23

Hönig, S. F., Kishimoto, M., Tristram, K. R. W., et al. 2013, ApJ, 771, 87

Ichikawa, K., Imanishi, M., Ueda, Y., et al. 2014, ApJ, 794, 139

Ichikawa, K., \& Inayoshi, K. 2017, ApJL, 840, L9

Ichikawa, K., Packham, C., Ramos Almeida, C., et al. 2015, ApJ, 803, 57

Ichikawa, K., Ricci, C., Ueda, Y., et al. 2017, ApJ, 835, 74

Ichikawa, K., Ueda, Y., Terashima, Y., et al. 2012, ApJ, 754, 45

Imanishi, M., Nakanishi, K., Izumi, T., \& Wada, K. 2018, ApJL, 853, L25

Inayoshi, K., \& Haiman, Z. 2016, ApJ, 828, 110

Isobe, T., Feigelson, E. D., \& Nelson, P. I. 1986, ApJ, 306, 490

Izumi, T., Kohno, K., Fathi, K., et al. 2017, ApJL, 845, L5

Jaffe, W., Meisenheimer, K., Röttgering, H. J. A., et al. 2004, Natur, 429, 47

Jun, H. D., Im, M., Lee, H. M., et al. 2015, ApJ, 806, 109

Kirkpatrick, A., Pope, A., Sajina, A., et al. 2015, ApJ, 814, 9

Kormendy, J., \& Ho, L. C. 2013, ARA\&A, 51, 511

Koss, M., Trakhtenbrot, B., Ricci, C., et al. 2017, ApJ, 850, 74

Koss, M. J., Assef, R., Baloković, M., et al. 2016, ApJ, 825, 85

Krabbe, A., Böker, T., \& Maiolino, R. 2001, ApJ, 557, 626

Krolik, J. H., \& Begelman, M. C. 1986, ApJL, 308, L55

Lamperti, I., Koss, M., Trakhtenbrot, B., et al. 2017, MNRAS, 467, 540

Lani, C., Netzer, H., \& Lutz, D. 2017, MNRAS, 471, 59

Lanz, L., Hickox, R. C., Balokovic, M., et al. 2018, arXiv:1811.02570

Lavalley, M., Isobe, T., \& Feigelson, E. 1992, adass I, 25, 245

Leftley, J. H., Tristram, K. R. W., Hönig, S. F., et al. 2018, AJ, 862, 17

López-Gonzaga, N., Burtscher, L., Tristram, K. R. W., Meisenheimer, K., \& Schartmann, M. 2016, A\&A, 591, A47

Lopez-Rodriguez, E., Alonso-Herrero, A., Diaz-Santos, T., et al. 2018, MNRAS, 478, 2350

Lopez-Rodriguez, E., Packham, C., Tadhunter, C., et al. 2014, ApJ, 793, 81

Lusso, E., Hennawi, J. F., Comastri, A., et al. 2013, ApJ, 777, 86

Lutz, D., Maiolino, R., Spoon, H. W. W., \& Moorwood, A. F. M. 2004, A\&A, 418, 46

Lutz, D., Shimizu, T., Davies, R. I., et al. 2018, A\&A, 609, 9

Lyu, J., Rieke, G. H., \& Alberts, S. 2016, ApJ, 816, 85

Lyu, J., \& Rieke, G. H. 2017, ApJ, 841, 76

Lyu, J., Rieke, G. H., \& Shi, Y. 2017, ApJ, 835, 257

Maiolino, R., Risaliti, G., Salvati, M., et al. 2010, A\&A, 517, A47

Maiolino, R., Shemmer, O., Imanishi, M., et al. 2007, A\&A, 468, 979
Marconi, A., Risaliti, G., Gilli, R., et al. 2004, MNRAS, 351, 169 Markowitz, A. G., Krumpe, M., \& Nikutta, R. 2014, MNRAS, 439, 1403

Martínez-Paredes, M., Aretxaga, I., Alonso-Herrero, A., et al. 2017, MNRAS, 468, 2

Masini, A., Comastri, A., Baloković, M., et al. 2016, A\&A, 589, A59

Mason, R. E., Lopez-Rodriguez, E., Packham, C., et al. 2012, AJ, 144, 11

Mateos, S., Carrera, F. J., Alonso-Herrero, A., et al. 2015, MNRAS, 449, 1422

Mateos, S., Carrera, F. J., Alonso-Herrero, A., et al. 2016, ApJ, 819, 166

Matsuoka, K., \& Woo, J.-H. 2015, ApJ, 807, 28

McLure, R. J., \& Dunlop, J. S. 2004, MNRAS, 352, 1390

Meléndez, M., Mushotzky, R. F., Shimizu, T. T., Barger, A. J., \& Cowie, L. L. 2014, ApJ, 794, 152

Mineo, S., Gilfanov, M., \& Sunyaev, R. 2012, MNRAS, 419, 2095

Minezaki, T., \& Matsushita, K. 2015, ApJ, 802, 98

Mullaney, J. R., Alexander, D. M., Goulding, A. D., \& Hickox, R. C. 2011, MNRAS, 414, 1082

Müller-Sánchez, F., Prieto, M. A., Mezcua, M., et al. 2013, ApJL, 763, L1

Murakami, H., Baba, H., Barthel, P., et al. 2007, PASJ, 59, S369

Murphy, E. J., Chary, R.-R., Dickinson, M., et al. 2011, ApJ, 732, 126

Mushotzky, R. F., Shimizu, T. T., Meléndez, M., \& Koss, M. 2014, ApJL, 781, L34

Nenkova, M., Sirocky, M. M., Ivezić, Ž., \& Elitzur, M. 2008a, ApJ, 685, 147

Nenkova, M., Sirocky, M. M., Nikutta, R., Ivezić, Ž., \& Elitzur, M. 2008b, ApJ, 685, 160

Netzer, H. 1987, MNRAS, 225, 55

Netzer, H. 2003, ApJL, 583, L5

Netzer, H. 2015, ARA\&A, 53, 365

Netzer, H., Lani, C., Nordon, R., et al. 2016, ApJ, 819, 123

Netzer, H., Lutz, D., Schweitzer, M., et al. 2007, ApJ, 666, 806

Novak, G. S. 2013, arXiv:1310.3833

Oh, K., Koss, M., Markwardt, C. B., et al. 2018, ApJS, 235, 4

Packham, C., Radomski, J. T., Roche, P. F., et al. 2005, ApJL, 618, L17

Poglitsch, A., Waelkens, C., Geis, N., et al. 2010, A\&A, 518, L2

Privon, G. C., Baum, S. A., O’Dea, C. P., et al. 2012, ApJ, 747, 46

Raban, D., Jaffe, W., Röttgering, H., Meisenheimer, K., \& Tristram, K. R. W 2009, MNRAS, 394, 1325

Radomski, J. T., Packham, C., Levenson, N. A., et al. 2008, ApJ, 681, 141

Ramos Almeida, C., Levenson, N. A., Alonso-Herrero, A., et al. 2011, ApJ, 731, 92

Ramos Almeida, C., Pérez García, A. M., Acosta-Pulido, J. A., \& Rodríguez Espinosa, J. M. 2007, AJ, 134, 2006

Ramos Almeida, C., \& Ricci, C. 2017, NatAs, 1, 679

Ricci, C., Bauer, F. E., Arevalo, P., et al. 2016, AJ, 820, 5

Ricci, C., Paltani, S., Awaki, H., et al. 2013, A\&A, 553, A29

Ricci, C., Trakhtenbrot, B., Koss, M. J., et al. 2017a, Natur, 549, 488

Ricci, C., Trakhtenbrot, B., Koss, M. J., et al. 2017b, ApJS, 233, 17

Ricci, C., Ueda, Y., Koss, M. J., et al. 2015, ApJL, 815, L13

Richards, G. T., Lacy, M., Storrie-Lombardi, L. J., et al. 2006, ApJS, 166, 470

Risaliti, G., Elvis, M., Fabbiano, G., et al. 2007, ApJL, 659, L111

Risaliti, G., Elvis, M., \& Gilli, R. 2002, ApJL, 566, L67

Risaliti, G., Nardini, E., Salvati, M., et al. 2011, MNRAS, 410, 1027

Rodriguez Espinosa, J. M., Rudy, R. J., \& Jones, B. 1987, ApJ, 312, 555

Rosario, D. J., Burtscher, L., Davies, R. I., et al. 2018, MNRAS, 473, 5658

Rosario, D. J., Santini, P., Lutz, D., et al. 2012, A\&A, 545, A45

Sajina, A., Yan, L., Armus, L., et al. 2007, ApJ, 664, 713

Shimizu, T. T., Meléndez, M., Mushotzky, R. F., et al. 2016, MNRAS, 456, 3335

Shimizu, T. T., Mushotzky, R. F., Meléndez, M., et al. 2017, MNRAS, 466, 3161 Simpson, C. 2005, MNRAS, 360, 565

Stalevski, M., Ricci, C., Ueda, Y., et al. 2016, MNRAS, 458, 2288

Stanley, F., Harrison, C. M., Alexander, D. M., et al. 2015, MNRAS, 453, 591

Symeonidis, M., Giblin, B. M., Page, M. J., et al. 2016, MNRAS, 459, 257

Trakhtenbrot, B. 2014, ApJL, 789, L9

Tran, Q. D., Lutz, D., Genzel, R., et al. 2001, ApJ, 552, 527

Treister, E., Krolik, J. H., \& Dullemond, C. 2008, ApJ, 679, 140

Tristram, K. R. W., Burtscher, L., Jaffe, W., et al. 2014, A\&A, 563, A82

Tueller, J., Mushotzky, R. F., Barthelmy, S., et al. 2008, ApJ, 681, 113

Ueda, Y., Akiyama, M., Hasinger, G., Miyaji, T., \& Watson, M. G. 2014, ApJ, 786, 104

Ueda, Y., Akiyama, M., Ohta, K., \& Miyaji, T. 2003, ApJ, 598, 886

Ueda, Y., Hiroi, K., Isobe, N., et al. 2011, PASJ, 63, S937

Wright, E. L., Eisenhardt, P. R. M., Mainzer, A. K., et al. 2010, AJ, 140, 1868

Xu, L., Rieke, G. H., Egami, E., et al. 2015, ApJS, 219, 18

Yu, Q., \& Tremaine, S. 2002, MNRAS, 335, 965 